\title{
Long noncoding RNAs, ENST00000598996 and ENST00000524265, are correlated with favorable prognosis and act as potential tumor suppressors in bladder cancer
}

\author{
CHONG SHEN ${ }^{1,2^{*}}$, YUJIE WANG ${ }^{1,2^{*}}$, ZHOULIANG WU $^{1,2^{*}}$, LA DA $^{1,2}$, SHEN GAO $^{1,2}$, LINGUO XIE $^{1,2}$, \\ YUNKAI QIE ${ }^{1,2}$, YINLEI WANG ${ }^{1,2}$, ZHE ZHANG $^{1,2}$, DAWEI TIAN ${ }^{1,2}$ and HAILONG HU ${ }^{1,2}$ \\ ${ }^{1}$ Department of Urology, The Second Hospital of Tianjin Medical University; ${ }^{2}$ Tianjin Key Laboratory of Urology, \\ Tianjin Institute of Urology, The Second Hospital of Tianjin Medical University, Tianjin 300211, P.R. China
}

Received November 20, 2019; Accepted June 4, 2020

DOI: $10.3892 / o r .2020 .7733$

\begin{abstract}
Bladder cancer (BC) is a serious malignancy worldwide due to its distant metastasis and high recurrence rates. Increasing evidence has indicated that dysregulated long non-coding RNAs (lncRNAs) are involved in tumorigenesis and progression in multiple malignancies. However, their clinical significances, biological functions and molecular mechanisms in $\mathrm{BC}$ remain poorly understood. Hence, the present study investigated the expression profile of IncRNAs and mRNAs in five $\mathrm{BC}$ tissues and the corresponding adjacent normal specimens using high-throughput RNA sequencing (RNA-seq). A total of 103 differentially expressed (DE) lncRNAs were identified, including 35 upregulated and 68 downregulated ones in BC tissues. Similarly, a total of 2,756 DE-mRNAs were detected, including 1,467 upregulated and 1,289 downregulated. Gene Ontology and Kyoto Encyclopedia of Genes and Genomes pathway analyses, and lncRNA-miRNA-mRNA network analyses suggested that these dysregulated IncRNAs are potentially implicated in the onset and progression of BC. Subsequently, four lncRNAs (upregulated ENST00000433108; downregulated ENST00000598996, ENST00000524265 and ENST00000398461) and two mRNAs (upregulated CCNB1 and CDK1) in 64 pairs of $\mathrm{BC}$ and adjacent normal tissues and four BC cell lines were detected using reverse transcription-quantitative PCR and these results were consistent with the sequencing data. Additionally, Fisher's exact test,
\end{abstract}

Correspondence to: Professor Hailong Hu or Professor Dawei Tian, Department of Urology, The Second Hospital of Tianjin Medical University, 23 Pingjiang Road, Jianshan Street, Hexi, Tianjin 300211, P.R. China

E-mail: hhllove2004@163.com

E-mail: jianshi001@126.com

${ }^{*}$ Contributed equally

Key words: bladder cancer, high-throughput RNA sequencing, long noncoding RNA, tumor suppressor, prognosis
Kaplan-Meier plots, and Cox regression analyses were used for elucidating the clinical values of ENST00000598996 and ENST00000524265. Furthermore, a receiver operating characteristic curve was constructed to assess their diagnostic values. The low expression level of ENST00000598996 and ENST00000524265 was correlated with unfavorable clinicopathological parameters, and shorter progression-free and overall survival time, whereas, ENST00000433108 was not associated with either. The in vitro functional experiments also revealed that the overexpression of ENST00000598996 and ENST00000524265 decreased the proliferation, migration, and invasion abilities of BC cells. Collectively, the results of the present study provide a novel landscape of IncRNA and mRNA expression profiles in BC. In addition, the results also indicated that ENST00000598996 and ENST00000524265 may serve as tumor suppressors, potential diagnostic biomarkers and prognostic predictors for patients with $\mathrm{BC}$.

\section{Introduction}

As one of the most frequently diagnosed urological malignancies of the urinary system, bladder cancer (BC) is also the 10th most commonly occurring type of cancer worldwide (1) and ranks 11th in terms of mortality (2). Despite advances in the treatment for $\mathrm{BC}$, including adjuvant chemoradiotherapies and surgical techniques, the recurrence and progression rates of $\mathrm{BC}$ remain high, and the quality of life and 5-year survival rates of $\mathrm{BC}$ exhibit no signs of significant improvement (3-7). Consequently, it is still urgent to investigate the underlying molecular mechanisms, new prognostic and predictive biomarkers of malignant progression and metastasis of bladder cancer.

Long non-coding RNAs (IncRNA), usually more than 200 bases in length, have become a new field of research (8-10). Compared with microRNAs (miRNAs) and protein-coding messenger RNAs (mRNAs), IncRNAs, owing to the greater tissue specificity, can be used for novel biomarkers of cancer diagnosis and prognosis (11). Recently, emerging studies have demonstrated that IncRNAs act as key regulators through their involvement in gene modulation at the transcriptional, post-transcriptional and chromosomal levels $(9,12,13)$. 
Furthermore, numerous studies have also revealed that lncRNAs play significant roles as oncogenes or suppressors in the carcinogenesis and evolution of various types of human malignancies, including colorectal $(14,15)$, hepatocellular $(16,17)$, gastric (18), breast (11), esophageal (19) and small-cell lung (20) cancers. Notably, several lncRNAs, such as DGCR5 (21), FAM83H-AS1 (22), GAS6-AS2 (23), HCG18 (24) and SNHG16 (25) have served as critical regulators in the biological behavior processes of BC over the past decades. For instance, overexpression of lncRNA-ATB markedly facilitated cell viability, migration, and invasion in bladder cancer cells and it may provide a potential prognostic biomarker and a therapeutic target for bladder cancer (26). Chen et al demonstrated that IncRNA-LNMAT1 accelerated lymphatic metastasis of bladder cancer via CCL2-dependent macrophage recruitment, and that it could serve as a potential therapeutic target for clinical intervention in LN-metastatic bladder cancer (12). Furthermore, lncRNAs could also act as anti-oncogenes. Tuo et al argued that lncRNA TP73-AS1 may serve as a tumor suppressor that participates in bladder cancer progression by inhibiting the epithelial-mesenchymal transition (EMT) process (27).

Appreciable progress has been made to comprehend lncRNA biology for the past few years, but the expression pattern, clinical value for $\mathrm{BC}$ diagnosis, therapy, and prognosis, as well as the possible molecular mechanisms of lncRNAs in $\mathrm{BC}$ remain largely unknown and warrant further study. Thus, the present study performed an investigation into lncRNAs and mRNAs in five pairs of BC and adjacent non-tumor tissues by RNA-sequencing (RNA-seq) in order to improve the current understanding of $\mathrm{BC}$ pathogenesis or development, and identified 103 markedly differentially expressed (DE) lncRNAs, and 2,756 significantly dysregulated mRNAs. Based on a bioinformatics analysis, the present study focused on the four IncRNAs (upregulated ENST00000433108; downregulated ENST00000598996, ENST00000524265 and ENST00000398461) and two mRNAs (namely CDK1 and CCNB1, both upregulated) in BC that are potentially associated with known pathogenic genes. These selected lncRNAs and mRNAs were validated via reverse transcription-quantitative PCR (RT-qPCR), and their dysregulation was conspicuously associated with certain clinicopathological characteristics and a worse prognosis in patients with BC. Furthermore, the present study also analyzed the sensitivity and specificity of these lncRNAs in the diagnosis of BC by constructing a ROC curve. According to the bioinformatics analysis, the screened lncRNAs have a competing endogenous (ce) RNA network regulation relationship with a variety of tumor-associated genes obtained from sequencing data of mRNAs; involving diverse tumor-associated signaling pathways, and then suggesting that these IncRNAs may be closely associated with BC progression through the aforementioned pathways. Notably, ectopic expression of ENST00000598996 or ENST00000524265 significantly suppressed cell migration, invasion and proliferation of BC cells.

The present study profiled the expression of lncRNAs and mRNAs in BC tissues and matched normal tissues, and examined the fundamental functional phenotypes of strictly selected lncRNAs (ENST00000598996 and ENST00000524265) in BC cells, with the aim of improving the current understanding of tumorigenesis and progression, but also to facilitate the prognosis prediction and clinical treatment management of $\mathrm{BC}$.

\section{Materials and methods}

Clinical specimens. A total of $64 \mathrm{BC}$ and matched non-tumorous tissues were collected from patients with $\mathrm{BC}$ who had undergone surgical resection without preoperative radiotherapy or chemotherapy at the Department of Urology of the Second Affiliated Hospital of Tianjin Medical University (China) between November 2015 and June 2019. The final diagnosis of each patient was determined histopathologically. The detailed clinical information of these patients is listed in Table SI. Overall survival (OS) was updated on 1 October, 2019 and was defined as the time from recruitment to death for any reason. Progression-free survival (PFS) was defined as the time from inclusion to recurrence or metastasis progression. In the present study, written informed consent was obtained from every patient or their guardians based on the guidelines approved by the Medical Ethics Committee of the Second Hospital of Tianjin Medical University.

Cell culture and transfection. Human normal bladder epithelium immortalized cells SV-HUC-1 and four bladder cancer cell lines (EJ-1, HTB-9, T24 and 253J-BV) were purchased from the Chinese Academy of Sciences Cell Bank (Shanghai, China); and cell lines were authenticated using STR DNA profiling. The EJ-1, HTB-9, T24, and 253J-BV cells were cultured in the RPMI-1640 medium (Biological Industries) supplemented with $10 \%$ fetal bovine serum (FBS) and $100 \mathrm{U} / \mathrm{ml}$ of penicillin, $100 \mathrm{mg} / \mathrm{ml}$ of streptomycin in a humidified incubator containing $5 \% \mathrm{CO}_{2}$ at $37^{\circ} \mathrm{C}$. The SV-HUC-1 cell line was cultured in the Ham's F-12K Nutrient Mixture (Gibco; Thermo Fisher Scientific, Inc.) with the same aforementioned culture conditions.

The ENST00000598996 and ENST00000524265 overexpression plasmid and the corresponding empty vectors (NC) were synthesized by Hanbio Biotechnology Co., Ltd. The Lipofectamine $^{\circledR} 2000$ Reagent (Invitrogen; Thermo Fisher Scientific, Inc.) was used for transfection of the aforementioned plasmids. After $24 \mathrm{~h}$ of plating, transfection was performed in serum-free medium using Lipofectamine ${ }^{\circledR} 2000$. After $6 \mathrm{~h}$, the transfected cells were placed in complete medium (RPMI-1640) containing $10 \% \mathrm{FBS}$ and maintained at $37^{\circ} \mathrm{C}$ in $5 \% \mathrm{CO}_{2}$.

$R N A$ extraction and quality control $(Q C)$. Total RNA was isolated from the bladder cancer and adjacent normal bladder tissues using TRIzol ${ }^{\circledR}$ reagent (Ambion) or an HP Total RNA kit (Omega Bio-Tek, Inc.) according to the manufacturer's protocol. RNA concentration and purity for tissues of sequencing analysis and subsequent verification was measured using a NanoDrop ND-1000 (Thermo Fisher Scientific, Inc.). In the present study, all RNA samples passed the quality standard based on a qualified ratio of optical density (OD) 260 to OD280 (1.8-2.1). Briefly based on denaturing agarose gel electrophoresis, RNA integrity and quality for tissues of sequencing analysis were validated by the presence of sharp clear bands of $28 \mathrm{~S}$ and $18 \mathrm{~S}$ ribosomal RNA, with a $28 \mathrm{~S}: 18 \mathrm{~S}$ ratio of $2: 1$, along with the absence of genomic DNA and degraded RNA.

High-throughput sequencing. First, ribosomal (r)RNAs were removed from the total RNA using Ribo-Zero rRNA 
Removal kits (Illumina, Inc.) according to the manufacturer's instructions. RNA libraries were constructed using a TruSeq Stranded Total RNA Library Prep kit (Illumina, Inc.) from the rRNA-depleted RNA based on the manufacturer's protocols. In the libraries, RNA quality and quantity were controlled using the BioAnalyzer 2100 system (Agilent Technologies; Thermo Fisher Scientific, Inc.). Subsequently, libraries were denatured to single-stranded DNA molecules, captured on Illumina flow cells, amplified in situ as clusters and finally sequenced for 150 cycles utilizing an Illumina HiSeq Sequencer according to the manufacturer's protocols.

Sequencing analysis of lncRNAs and mRNAs. The original reads were obtained from the Illumina HiSeq 4000 sequencer and 3 ' adaptor-trimming and removal of low-quality reads were performed using Cutadapt software (v1.9.3) (https://cutadapt. readthedocs.io/en/stable/). The resulting high-quality clean reads were utilized to analyze 1 chNAs and mRNAs. The high-quality reads were aligned to the human reference genome (UCSC HG19) using HISAT2 software (https://ccb. jhu.edu/software/hisat2).

Identification of DE lncRNAs and mRNAs and RT-qPCR validation. Next, guided by the Ensembl GTF gene annotation file (http://ftp.ensembl.org/pub/release-75/gtf/homo_sapiens/), Cuffdiff software (v2.2.1, part of cufflinks) (http://cole-trapnell-lab.github.io/cufflinks/cuffdiff/index.html) was used to obtain the FPKM as the expression profiles of lncRNAs and mRNAs, and the fold change (FC) and P-values were calculated based on the FPKM; differentially expressed lncRNAs and mRNAs $(F C \geq 2.0$ and P-values $\leq 0.05)$ were classified as significant. Scatter plots were drawn to reveal the different lncRNA or mRNA expression patterns in the BC and matched adjacent non-cancerous specimens by the CRAN packages ggplot2 in R (https://www.r-project.org/).

RT-qPCR was performed to validate the expression of the selected DE lncRNAs and mRNAs from RNA sequencing. The qPCR thermocycling conditions were as follows: Initial denaturation at $95^{\circ} \mathrm{C}$ for $10 \mathrm{~min}$, followed by 40 cycles at $95^{\circ} \mathrm{C}$ for $10 \mathrm{sec}$ and $60^{\circ} \mathrm{C}$ for $50 \mathrm{sec}$. Following the manufacturer's instructions, total RNA was extracted using TRIzol ${ }^{\circledR}$ reagent (Ambion) or an HP Total RNA kit (Omega Bio-Tek, Inc.) and then reverse-transcribed by Transcriptor First Strand cDNA Synthesis kit and amplified in triplicate on an Applied Biosystems 7500 Fast Real-Time PCR System (Applied Biosystems; Thermo Fisher Scientific, Inc.) using the FastStart Universal SYBR Green Master with ROX (Roche Diagnostics). GAPDH was used as an internal reference for normalization. The relative expression ratio of these lncRNAs and mRNAs was calculated through the $2^{-\Delta \Delta \mathrm{Cq}}$ method (28). All primers were synthesized by Sangon Biotech Co. Ltd. and the sequences are presented in Table I.

Bioinformatic analyses. Gene Ontology (GO) and Kyoto Encyclopedia of Genes and Genomes (KEGG) analyses were performed in order to determine the underlying functions for the differentially expressed lncRNA-associated intersection genes (downstream mRNAs predicted by lncRNAs vs. sequencing DE mRNAs) using the Database for Annotation, Visualization, and Integrated Discovery (DAVID) Bioinformatics resources (http://david.abcc.ncifcrf.gov/) (29). GO analysis can be classified into biological processes (BP), cellular components (CC), and molecular functions (MF). $\mathrm{P}<0.05$ was considered to indicate a statistically significant difference.

Construction of the ceRNA and protein-protein interaction (PPI) networks. For determining the interactions between mRNAs and lncRNAs, the IncRNA-miRNA-mRNA network was constructed. The miRanda (http://www.microrna. org/microrna/home.do) and DIANA (http://carolina.imis. athena-innovation.gr/) (30) tools were utilized to predict the first top 5 miRNAs of the screened six DE lncRNAs. Subsequently, the miRTarBase (http://mirtarbase.mbc.nctu.edu.tw/) (31) and TargetScan (http://www.targetscan.org/) (32) databases were performed to predict the miRNA-targeted mRNAs, and an intersection with sequencing DE mRNAs was constructed.

Then, according to these intersection genes, the PPI network was performed using STRING (version 11.0) (https://string-db. org/) online analysis. The construction and visualization of the IncRNA-miRNA-mRNA and PPI networks were accomplished by Cytoscape v3.6.1 (http://www.cytoscape.org/).

Cell proliferation assay. Cell proliferation was investigated using a Cell Counting Kit-8 (CCK-8) and colony-formation experiments. For the CCK- 8 assay, $2 \times 10^{3}$ cells/well were seeded into 96 -well plates. At the indicated time-points $(0,24$, 48,72 and $96 \mathrm{~h}$ ), each well had $10 \mu \mathrm{lCCK}-8$ solution (Beyotime Institute of Biotechnology) added, and the plates were incubated at $37^{\circ} \mathrm{C}$ for another $3.5 \mathrm{~h}$ in the dark. The absorbance was measured at a wavelength of $450 \mathrm{~nm}$ using a microplate reader. For the clone formation assay, cells (500/well) were seeded into 6-well plates and cultured for 10 days. Next, the cell colonies with a diameter $>1 \mathrm{~mm}$ were stained with $0.1 \%$ crystal violet at room temperature for $15 \mathrm{~min}$ and counted.

Cell migration and invasion assays. Wound healing and Transwell assays (without Matrigel coating) were performed to evaluate cell migration. The Transwell assay [with Matrigel coating (BD Biosciences)] was performed to assess cell invasion. For the wound healing assay, transfected cells were cultured to $90-95 \%$ confluency in 6-well plates and scratched using 200- $\mu$ l pipette tips. Migration images were captured at 0 and $36 \mathrm{~h}$ after scraping with an inverted microscope (magnification x200; Olympus Corporation) and analyzed objectively via Image J v1.46 software (National Institutes of Health). For the Transwell assay, $5 \times 10^{4}$ transfected cells in FBS-free RPMI-1640 medium were seeded into the top chamber, while the lower chamber was supplemented with RPMI-1640 medium containing 20\% FBS. After culturing for $36 \mathrm{~h}$, the non-migrated or non-invasive cells were removed with a cotton tip, and the remaining cells on the bottom surface were fixed with $4 \%$ paraformaldehyde at room temperature for $15 \mathrm{~min}$ and stained with $0.1 \%$ crystal violet for $15 \mathrm{~min}$ at room temperature. The stained cells were imaged in five randomly selected fields under an optical microscope (magnification x400; Olympus Corporation) and counted using ImageJ v1.46 software (National Institutes of Health).

Statistical analysis. Statistical analyses were performed using GraphPad Prism 7.0 software (GraphPad Software, Inc.) and 
Table I. Primers used to validate lncRNA and mRNA expression.

\begin{tabular}{|c|c|c|c|}
\hline Gene name & Primer type & Primer sequence & Product length (bp) \\
\hline \multirow[t]{2}{*}{ ENST00000433108 } & Forward & 5'-AGGGGCAAAAGAAACATCCAA-3' & \multirow[t]{2}{*}{237} \\
\hline & Reverse & 5'-CCCCTGATCTAGCCACTCATC-3' & \\
\hline \multirow[t]{2}{*}{ ENST00000398461 } & Forward & 5'-GCCCTAGGGGAGTGACTACA-3' & \multirow[t]{2}{*}{155} \\
\hline & Reverse & 5'-CCCCTGTGCCCAATTTCAAC-3' & \\
\hline \multirow[t]{2}{*}{ ENST00000524265 } & Forward & 5'-ACAAGAAGCAGAATGGGCCT-3' & \multirow[t]{2}{*}{192} \\
\hline & Reverse & 5'-AATCACACGGGCTTTGCATT-3' & \\
\hline \multirow[t]{2}{*}{ ENST00000598996 } & Forward & 5'-CCGGTCACTTCACGATGACA-3' & \multirow[t]{2}{*}{193} \\
\hline & Reverse & 5'-CAGGACCTCCAGGGAAACAC-3' & \\
\hline \multirow[t]{2}{*}{ CCNB1 } & Forward & 5'-AACTTTCGCCTGAGCCTATTTT-3' & \multirow[t]{2}{*}{228} \\
\hline & Reverse & 5'-TTGGTCTGACTGCTTGCTCTT-3' & \\
\hline \multirow[t]{2}{*}{ CDK1 } & Forward & 5'-AAACTACAGGTCAAGTGGTAGCC-3' & \multirow[t]{2}{*}{148} \\
\hline & Reverse & 5'-TCCTGCATAAGCACATCCTGA-3' & \\
\hline \multirow[t]{2}{*}{ GAPDH } & Forward & 5'-CGGAGTCAACGGATTTGGTC-3' & \multirow[t]{2}{*}{180} \\
\hline & Reverse & 5'-TTCCCGTTCTCAGCCTTGAC-3' & \\
\hline
\end{tabular}

lncRNA, long non-coding RNA; mRNA, messenger RNA.

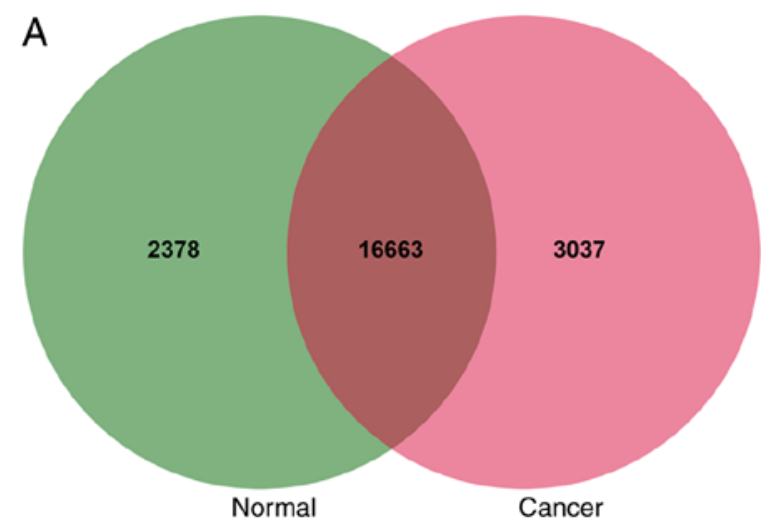

C

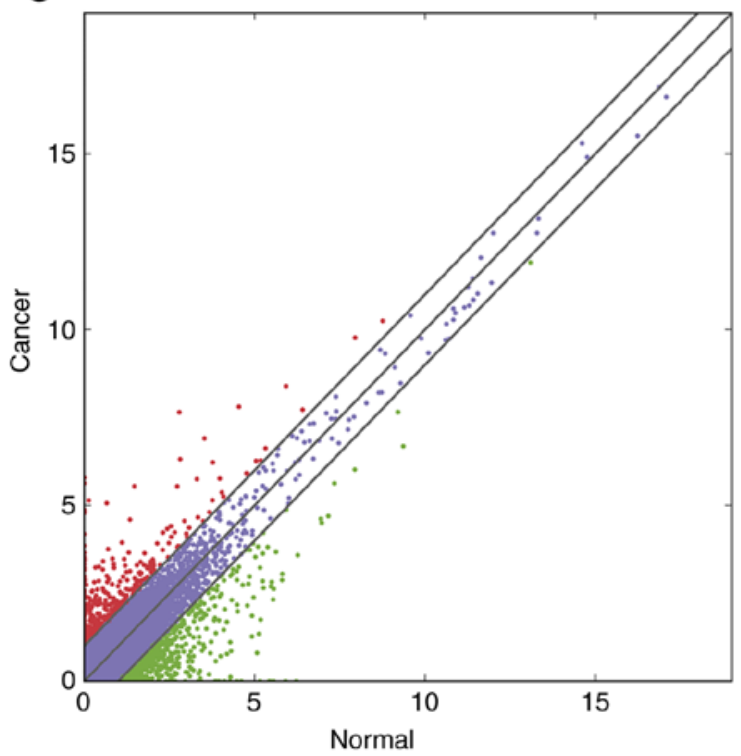

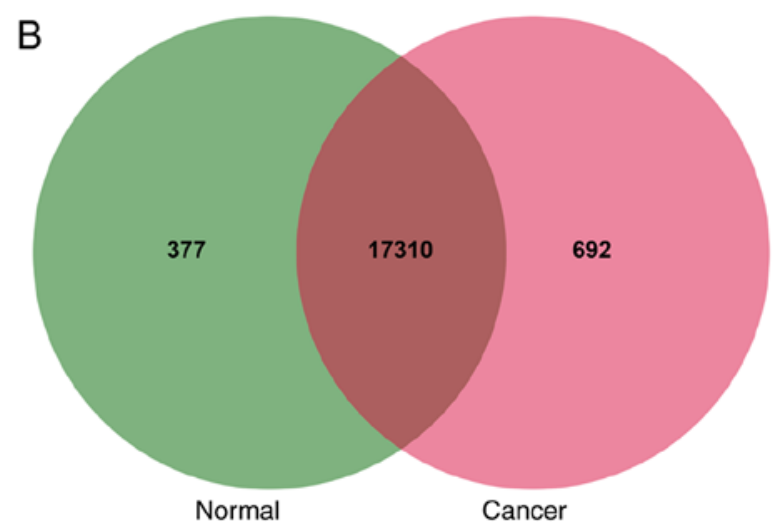

D

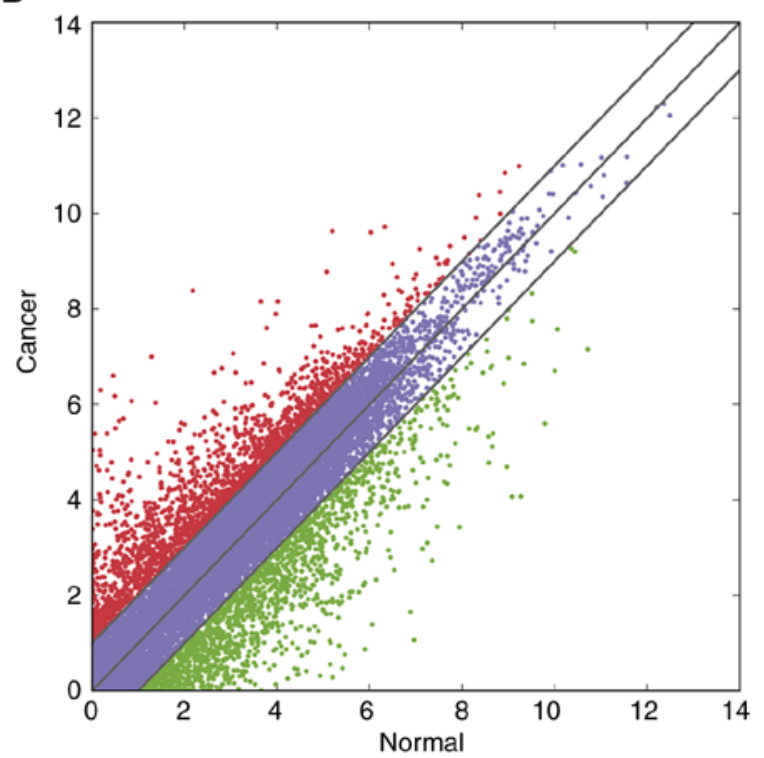

Figure 1. (A and B) Venn diagrams revealed the number of overlapping lncRNAs or mRNAs between the BC (marked as 'Cancer') and adjacent non-tumor tissues ('Control'). (C and D) Scatter plots representing the up- and downregulated lncRNAs or mRNAs in BC tissues vs. controls (log2 FC $\geq 1$ or $\leq-1$; $\mathrm{P}<0.05$ ). Red discs on the upper left corner represent upregulation; green discs on the lower right side represent downregulation; purple discs in the middle part represent no significant difference. lncRNA, long non-coding RNA; mRNA, messenger RNA; BC, bladder cancer. 
Table II. Upregulated and downregulated lncRNAs in whole are listed.

\begin{tabular}{|c|c|c|c|c|c|c|}
\hline Transcript ID & Regulation & $\log _{2} \mathrm{Fc}$ & P-value & Chr & Strand & Biotype \\
\hline ENST00000410910 & Up & $\inf$ & 0.01745 & chr2:17304107-17304407 & - & misc_RNA \\
\hline ENST00000434300 & Up & $\inf$ & 0.02275 & chr1:192486768-192536347 & - & antisense \\
\hline ENST00000438158 & Up & $\inf$ & 0.04595 & chr1:222215046-222238105 & - & lincRNA \\
\hline ENST00000522365 & Up & $\inf$ & 0.0214 & chr8:84315992-84321135 & + & lincRNA \\
\hline ENST00000538428 & Up & $\inf$ & 0.02985 & chr1:98453555-98515419 & - & lincRNA \\
\hline ENST00000548280 & Up & $\inf$ & 0.03395 & chr14:28787346-28887818 & + & lincRNA \\
\hline ENST00000566101 & Up & $\inf$ & 0.00985 & chr18:37646249-37679197 & + & lincRNA \\
\hline ENST00000566876 & Up & $\inf$ & 0.00765 & chr16:50424826-50429044 & - & lincRNA \\
\hline ENST00000602912 & Up & $\inf$ & 0.0165 & chr11:67227338-67227784 & + & lincRNA \\
\hline TCONS_00017351 & Up & $\inf$ & 0.0294 & chrX:86979060-86983512 & - & long_nonc. \\
\hline TCONS_00017630 & Up & $\inf$ & 0.01895 & chrY:7302523-7388562 & + & long_nonc. \\
\hline TCONS_00020860 & Up & $\inf$ & 0.0048 & chr12:88006397-88043289 & - & long_nonc. \\
\hline TCONS_00020861 & Up & $\inf$ & 0.00695 & chr12:88045658-88064325 & - & long_nonc. \\
\hline TCONS_00022565 & Up & $\inf$ & 0.0313 & chr14:80931125-80938379 & + & long_nonc. \\
\hline TCONS_12_00030270 & Up & $\inf$ & 0.0043 & chrX:97098491-97101678 & + & long_nonc. \\
\hline TCONS_12_00005321 & Up & 147.61 & 0.013 & chr11:65184125-65213011 & + & long_nonc. \\
\hline TCONS_00001651 & Up & 5.08 & 0.00025 & chr1:121484056-121485434 & - & long_nonc. \\
\hline uc001ycm.1 & Up & 3.70 & 0.0328 & chr14:94571181-94583033 & + & long_nonc. \\
\hline ENST00000429829 & Up & 3.57 & 0.01185 & chrX:73012039-73072588 & - & lincRNA \\
\hline ENST00000562848 & Up & 3.39 & 0.04505 & chr12:54375292-54449814 & + & lincRNA \\
\hline TCONS_12_00027748 & Up & 3.17 & 0.04745 & chr8:49277961-49337122 & + & long_nonc. \\
\hline ENST00000525217 & Up & 3.02 & 0.0245 & chr11:287304-288987 & + & antisense \\
\hline ENST00000587187 & Up & 3.01 & 0.0078 & chr19:4903091-4962165 & + & processed_tr. \\
\hline ENST00000448587 & Up & 2.75 & 0.0105 & chr19:5558177-5581226 & - & lincRNA \\
\hline ENST00000605886 & Up & 2.47 & 0.0232 & chr1:156611181-156631216 & - & antisense \\
\hline ENST00000570148 & Up & 2.00 & 0.01645 & chr16:81344335-81347028 & - & antisense \\
\hline TCONS_00006377 & Up & 1.73 & 0.00415 & chr3:195366860-195378976 & + & long_nonc. \\
\hline TCONS_12_00025984 & Up & 1.67 & 0.02905 & chr7:65300346-65310515 & + & long_nonc. \\
\hline ENST00000439156 & Up & 1.65 & 0.01925 & chr1:61005920-61106163 & - & lincRNA \\
\hline ENST00000400385 & Up & 1.65 & 0.0472 & chr21:45225638-45232448 & - & antisense \\
\hline ENST00000606434 & Up & 1.62 & 0.0259 & chr5:6671218-6674499 & + & lincRNA \\
\hline TCONS_12_00025849 & Up & 1.59 & 0.0122 & chr7:45831387-45863181 & + & long_nonc. \\
\hline NR_104643 & Up & 1.51 & 0.01445 & chr5:42985500-42993435 & - & long_nonc. \\
\hline ENST00000566699 & Up & 1.48 & 0.04175 & chr7:139877060-139879440 & + & antisense \\
\hline ENST00000433108 & Up & 1.33 & 0.01015 & chr1:209602164-209606183 & + & lincRNA \\
\hline TCONS_00006771 & Up & 1.24 & 0.0104 & chr3:197360473-197374168 & - & long_nonc. \\
\hline GU228573 & Down & -52.13 & 0.02865 & chr7:55086713-55324313 & + & long_nonc. \\
\hline uc021xrd.1 & Down & -5.44 & 0.0098 & chr4:119809995-119982402 & + & long_nonc. \\
\hline ENST00000474768 & Down & -5.24 & 0.01835 & chr3:64501332-64997143 & + & antisense \\
\hline ENST00000433310 & Down & -5.11 & 0.00005 & chr21:29811666-30047170 & - & lincRNA \\
\hline ENST00000460833 & Down & -5.10 & 0.00625 & chr3:64501332-64997143 & + & antisense \\
\hline ENST00000481312 & Down & -5.09 & 0.0287 & chr3:64501332-64997143 & + & antisense \\
\hline ENST00000555383 & Down & -4.80 & 0.0094 & chr14:96671015-96735304 & + & sense_intr. \\
\hline ENST00000515734 & Down & -4.77 & 0.03565 & chr5:58264864-59843484 & + & lincRNA \\
\hline ENST00000593604 & Down & -4.54 & 0.02775 & chr16:86508134-86542705 & - & lincRNA \\
\hline ENST00000598996 & Down & -4.53 & 0.0471 & chr16:86508134-86542705 & - & lincRNA \\
\hline ENST00000426585 & Down & -4.47 & 0.03795 & chr22:17082776-17185367 & + & lincRNA \\
\hline ENST00000608684 & Down & -4.46 & 0.0486 & chr1:78444858-78604133 & - & antisense \\
\hline ENST00000602478 & Down & -4.37 & 0.00165 & chr22:43011249-43011913 & + & lincRNA \\
\hline ENST00000398460 & Down & -4.18 & 0.0053 & chr14:101245746-101327368 & + & lincRNA \\
\hline uc022buq.1 & Down & -4.02 & 0.0404 & chrX:31115793-33357558 & - & long_nonc. \\
\hline ENST00000562393 & Down & -4.00 & 0.0376 & chr16:84853589-84954374 & + & long_nonc. \\
\hline
\end{tabular}


Table II. Continued.

\begin{tabular}{|c|c|c|c|c|c|c|}
\hline Transcript ID & Regulation & $\log _{2} \mathrm{Fc}$ & P-value & Chr & Strand & Biotype \\
\hline AL049990 & Down & -3.99 & 0.0392 & chr4:121952575-121954272 & - & long_nonc. \\
\hline ENST00000522358 & Down & -3.89 & 0.00175 & chr5:148786251-148812399 & + & lincRNA \\
\hline uc021pwm.1 & Down & -3.88 & 0.00725 & chr10:97071527-97321171 & - & long_nonc. \\
\hline ENST00000421206 & Down & -3.87 & 0.0343 & chr10:120967100-121215131 & + & sense_intr. \\
\hline antipeg11_dna & Down & -3.87 & 0.00005 & chr14:101346991-101351184 & + & long_nonc. \\
\hline ENST00000398878 & Down & -3.52 & 0.004 & chr16:29262828-30215631 & + & lincRNA \\
\hline uc003bgk.1 & Down & -3.50 & 0.0189 & chr22:45898117-45998697 & + & long_nonc. \\
\hline ENST00000604430 & Down & -3.40 & 0.02785 & chr16:29262828-30215631 & + & lincRNA \\
\hline ENST00000445466 & Down & -3.38 & 0.02205 & chr3:151961616-152214602 & - & antisense \\
\hline ENST00000505254 & Down & -3.20 & 0.01775 & chr5:148786251-148812399 & + & lincRNA \\
\hline ENST00000415513 & Down & -3.10 & 0.0329 & chr7:104581509-105039755 & + & lincRNA \\
\hline NR_024344 & Down & -3.10 & 0.0002 & chr11:133766329-133771635 & - & long_nonc. \\
\hline uc001rpy.3 & Down & -3.03 & 0.03735 & chr12:48099867-48231681 & - & long_nonc. \\
\hline uc002vgv.1 & Down & -2.99 & 0.0171 & chr2:218664511-218867718 & - & long_nonc. \\
\hline ENST00000573866 & Down & -2.95 & 0.00015 & chr17:19015312-19015949 & - & lincRNA \\
\hline ENST00000452120 & Down & -2.92 & 0.00005 & chr14:101245746-101327368 & + & lincRNA \\
\hline U54776 & Down & -2.88 & 0.00045 & chr6:136172833-136546762 & + & long_nonc. \\
\hline ENST00000524265 & Down & -2.87 & 0.01335 & chr5:148786251-148812399 & + & lincRNA \\
\hline ENST00000398461 & Down & -2.87 & 0.00045 & chr14:101245746-101327368 & + & lincRNA \\
\hline TCONS_00024657 & Down & -2.79 & 0.0003 & chr16:53069601-53086785 & - & long_nonc. \\
\hline ENST00000443576 & Down & -2.73 & 0.04575 & chr13:33677271-33924767 & - & lincRNA \\
\hline ENST00000452320 & Down & -2.66 & 0.03175 & chr7:77646392-79100524 & + & processed_tr. \\
\hline ENST00000533633 & Down & -2.60 & 0.00005 & chr11:13983913-14295237 & + & processed_tr. \\
\hline NR_034108 & Down & -2.52 & 0.03375 & chr6:111620233-111927481 & + & long_nonc. \\
\hline ENST00000587762 & Down & -2.52 & 0.00005 & chr19:13945329-13947173 & - & lincRNA \\
\hline ENST00000604052 & Down & -2.52 & 0.0484 & chr2:28607275-28640179 & + & sense_intr. \\
\hline ENST00000571722 & Down & -2.49 & 0.0154 & chr17:18966761-18967449 & - & lincRNA \\
\hline ENST00000563424 & Down & -2.44 & 0.0017 & chr16:50679719-50683160 & + & lincRNA \\
\hline uc002aqh.1 & Down & -2.33 & 0.0031 & chr15:67152640-67156987 & + & long_nonc. \\
\hline uc002aqi.3 & Down & -2.14 & 0.0026 & chr15:67156988-67159361 & + & long_nonc. \\
\hline ENST00000608023 & Down & -2.02 & 0.0001 & chr1:207974862-208042495 & - & processed_tr. \\
\hline ENST00000568809 & Down & -2.00 & 0.0244 & chrX:99928326-99928978 & + & antisense \\
\hline uc001kct.3 & Down & -1.90 & 0.0163 & chr10:85926983-85931832 & - & long_nonc. \\
\hline NR_121662 & Down & -1.90 & 0.00015 & chr3:114033346-114866118 & - & long_nonc. \\
\hline AF052103 & Down & -1.80 & 0.02055 & chr16:85399033-85400346 & + & long_nonc. \\
\hline uc010yvs.2 & Down & -1.79 & 0.0181 & chr2:100889752-100939195 & - & long_nonc. \\
\hline TCONS_00026354 & Down & -1.76 & 0.03035 & chr18:66817066-66832387 & + & long_nonc. \\
\hline ciRS-7 & Down & -1.74 & 0.00035 & chrX:139863223-139866829 & + & long_nonc. \\
\hline TCONS_12_00017201 & Down & -1.72 & 0.04045 & chr21:33937195-33940382 & - & long_nonc. \\
\hline ENST00000606034 & Down & -1.70 & 0.0252 & chr1:931345-933431 & - & lincRNA \\
\hline ENST00000448490 & Down & -1.62 & 0.00395 & chr10:91589266-91600618 & + & lincRNA \\
\hline ENST00000432045 & Down & -1.53 & 0.01185 & chr7:130538478-130618587 & - & lincRNA \\
\hline ENST00000500358 & Down & -1.53 & 0.0482 & chr4:100010007-100222513 & + & antisense \\
\hline ENST00000565297 & Down & -1.51 & 0.0404 & chr8:79749763-79752757 & + & lincRNA \\
\hline ENST00000453660 & Down & -1.24 & 0.01495 & chr9:136125787-136150617 & - & processed_tr. \\
\hline ENST00000584058 & Down & -1.19 & 0.0355 & chr3:15708742-15901278 & + & misc_RNA \\
\hline ENST00000437646 & Down & -inf & 0.00005 & chr17:19322347-19327670 & + & lincRNA \\
\hline ENST00000446601 & Down & -inf & 0.0128 & $\operatorname{chr} 3: 26753797-26754855$ & + & lincRNA \\
\hline ENST00000592429 & Down & -inf & 0.04485 & chr19:5158505-5340814 & + & antisense \\
\hline TCONS_00006712 & Down & -inf & 0.0396 & chr3:179243601-179245572 & - & long_nonc. \\
\hline TCONS_00016239 & Down & -inf & 0.00525 & chr9:10095-10799 & - & long_nonc. \\
\hline TCONS_00022925 & Down & -inf & 0.0455 & chr14:24096369-24096959 & + & long_nonc. \\
\hline
\end{tabular}

lncRNA, long non-coding RNA; Fc, Fold change; inf, infinity; Chr, Chromosome; long_nonc., long_noncoding; sense_intr., sense_intronic; processed_tr., processed_transcript. 
SPSS 20.0 software (IBM Corp.). All data are presented as the mean \pm standard deviation of at least three independent experiments. $\mathrm{P}<0.05$ was considered to indicate a statistically significant difference.

A paired $t$-test was used to analyze the differences between $\mathrm{BC}$ tissues and paired normal tissues. Two-way analysis of variance (ANOVA) followed by Dunnett's post hoc test was implemented for comparisons of multiple groups, as noted in the Figs. 5C and 9A-E. The associations between ENST00000598996 or ENST00000524265 levels and clinicopathological parameters of patients with $\mathrm{BC}$ were further analyzed via a Fisher's exact test. A ROC curve was drawn to estimate diagnostic performance. Survival analysis was conducted by Kaplan-Meier method and log-rank test for significance in GraphPad Prism 7.0. Survival data were further evaluated using the univariate and multivariate Cox proportional hazards model.

\section{Results}

Overview of IncRNA and mRNA profiles in BC tissues. The present study first analyzed the profiling of lncRNAs and mRNAs in five paired human BC and adjacent non-cancerous tissues via RNA-Seq. A total of 37,684 lncRNAs and 20,266 mRNAs were detected in both groups. For lncRNAs, 2,378 were only detected in the paired control tissues, 3,037 were only detected in $\mathrm{BC}$ tissues and 16,663 were detected in both groups (Fig. 1A). Of the total 37,684 lncRNAs, 35 upregulated and 68 downregulated lncRNAs were significantly differentially expressed in $\mathrm{BC}$ specimens when compared with controls ( $\mathrm{P}<0.05$, fold change $\geq 2.0$ ) (Table II; Figs. $1 \mathrm{C}$ and 2A). Based on the sequencing data, there were more downregulated lncRNAs than upregulated. For mRNAs, 377 were only discovered in the matched control tissues, 692 were only detected in BC tissues and 17,310 were discovered in both groups (Fig. 1B). Among the whole 20,266 mRNAs, 1,467 upregulated and 1,289 downregulated mRNAs were significantly aberrantly expressed in $\mathrm{BC}$ tissues $(\mathrm{P}<0.05$, fold change $\geq 2.0$ ) (Figs. 1D and $2 \mathrm{~A}$ ).

Subsequently, the present study predicted their respective sponge miRNAs and target mRNAs based on the abnormally expressed lncRNA data. Next, the present study compared these mRNA predictions with the mRNA sequencing results (Fig. 2B). Hence, 236 targeted DE-mRNAs were identified, including 120 upregulated and 116 downregulated (Table SII). Then, the aforementioned resulting mRNAs (236) were utilized for the GO, KEGG pathway and ceRNA network analyses.

Functional analysis of miRNA target genes. It is well known that lncRNAs are crucial transcriptional regulators of gene expression involved in the nosogenesis of multiple types of cancer. In order to investigate the underlying mechanisms of dyseregulated lncRNAs in the carcinogenesis or development of $\mathrm{BC}, \mathrm{GO}$ enrichment analysis of the aforementioned 236 target DE-mRNAs was conducted to estimate the functional performance of the 103 DE lncRNAs.

GO enrichment analysis were classified as three aspects: $\mathrm{BP}, \mathrm{MF}$ and CC. The most enriched and meaningful BP were associated with 'gene regulation of apoptotic process' (GO:0042981), 'regulation of cell cycle' (GO:0051726), 'positive regulation of gene expression' (GO:0010628), 'positive
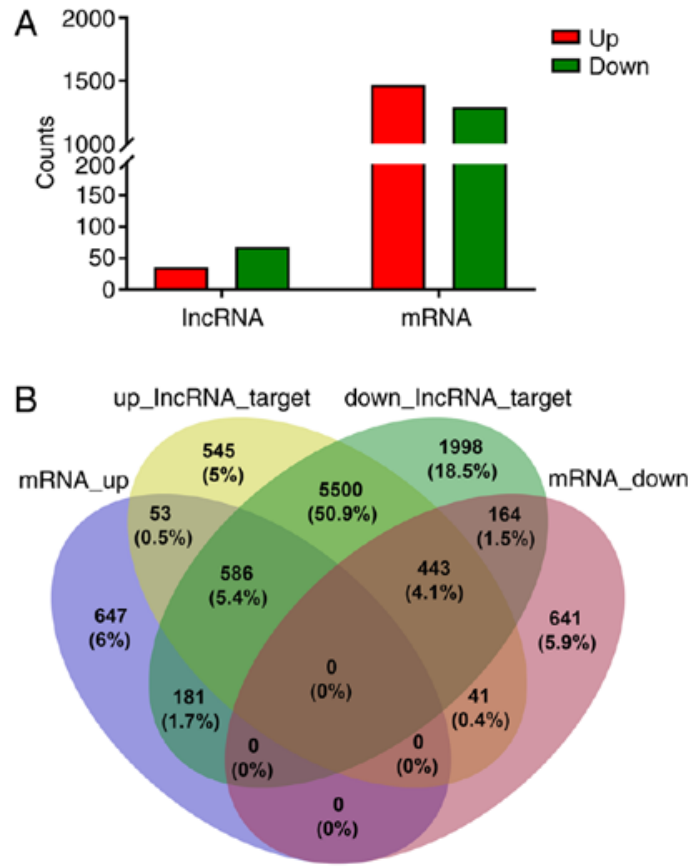

Figure 2. Number of DE 1ncRNAs and mRNAs in BC tissues when compared with controls. (A) Histogram representing the count of up- and downregulated lncRNAs and mRNAs in BC tissues. (B) Venn diagram indicates the overlapping counts of upregulated mRNAs (labeled as 'mRNA_up'), downregulated mRNAs ('mRNA_down'), targeted mRNAs in upregulated_lncRNAs ('up_lncRNA_target'), and targeted mRNAs in downregulated_lncRNAs ('down_lncRNA_target'). DE, differentially expressed; lncRNA, long non-coding RNA; mRNA, messenger RNA; BC, bladder cancer.

regulation of cell proliferation' (GO:0008284) and 'regulation of angiogenesis' (GO:0045765) (Fig. 3A). The most enriched GO terms under MF were 'DNA binding' (GO:0003677), 'protein kinase activity' (GO:0004672), 'ubiquitin-like protein ligase binding' (GO:0044389), 'MAP kinase phosphatase activity' (GO:0033549) and 'transcription regulatory region DNA binding' (GO:0044212) (Fig. 3B). In the CC analysis, 'nuclear chromosome part' (GO:0044454), 'chromosome, telomeric region' (GO:0000781), 'sarcoplasm' (GO:0016528), 'integral component of plasma membrane' (GO:0005887) and 'focal adhesion' (GO:0005925) were the significantly enriched terms (Fig. 3C).

Next, in order to identify the IncRNA indirectly-regulated signaling pathways, the present study performed a KEGG analysis on these 236 DE-mRNAs. The significantly enriched KEGG pathways are presented in Fig. 4A and B. Among them, 'pathways in cancer', 'cell cycle', 'MAPK signaling pathway', 'HIF-1 signaling pathway', 'PI3K-Akt signaling pathway', 'transcriptional misregulation in cancer', 'cAMP signaling pathway', 'p53 signaling pathway and NF- $\kappa B$ signaling pathway', were reported as various cancer-associated pathways. The results indicated that these pathways may significantly contribute to the pathogenesis and development of BC.

$R T-q P C R$ verification of $\operatorname{lnc} R N A$ and $m R N A$ expression. Subsequently, to confirm the reliability and validity of the aforementioned analyses, the present study randomly selected four lncRNAs (upregulated ENST00000433108; downregulated ENST00000598996, ENST00000524265 and 
A

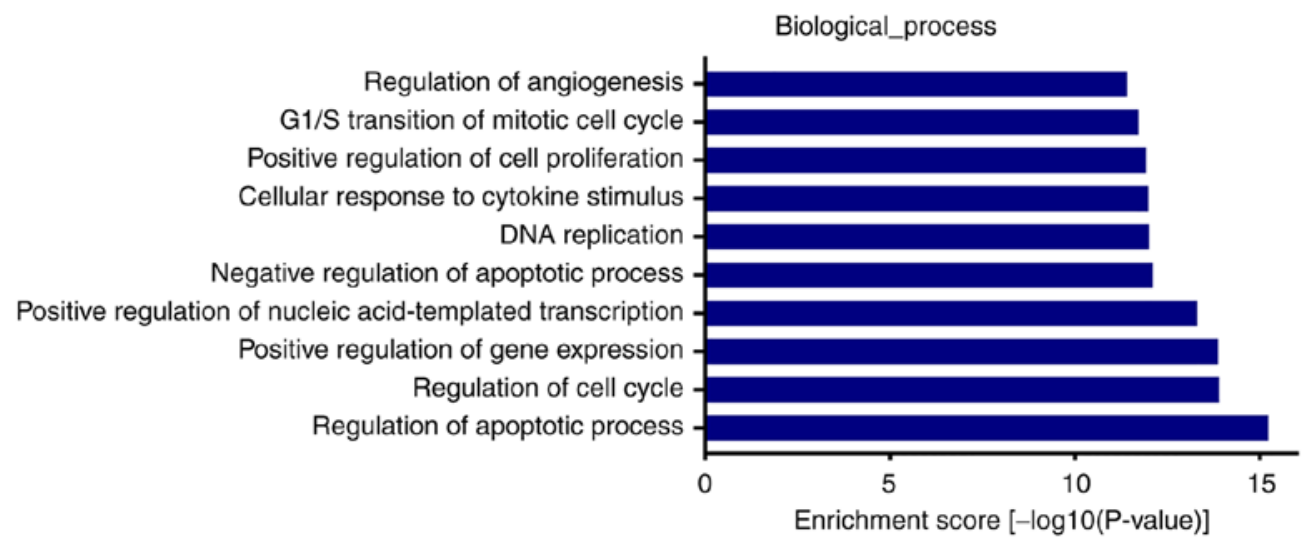

B

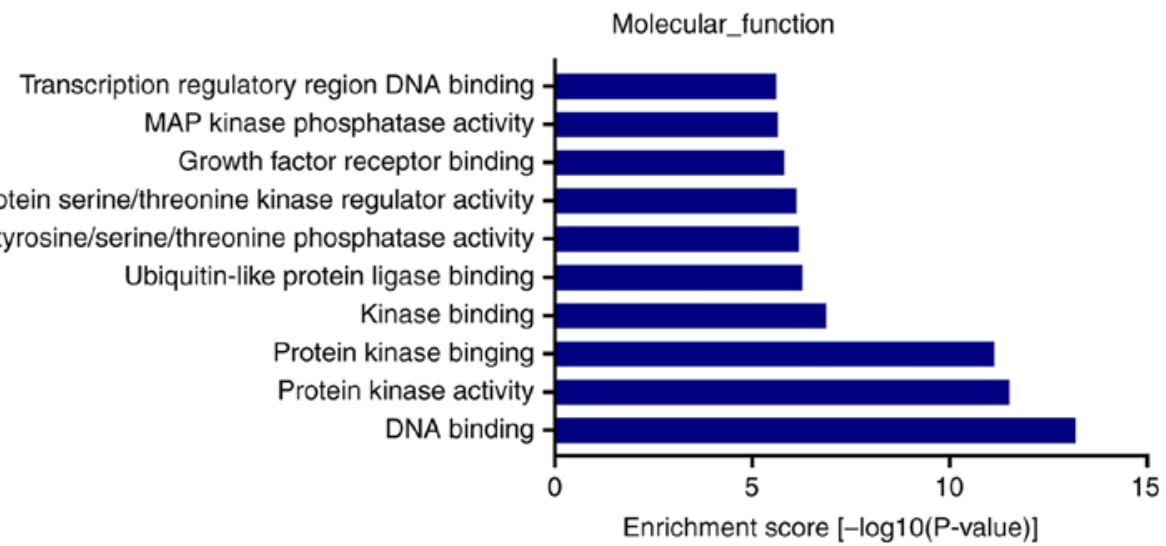

C

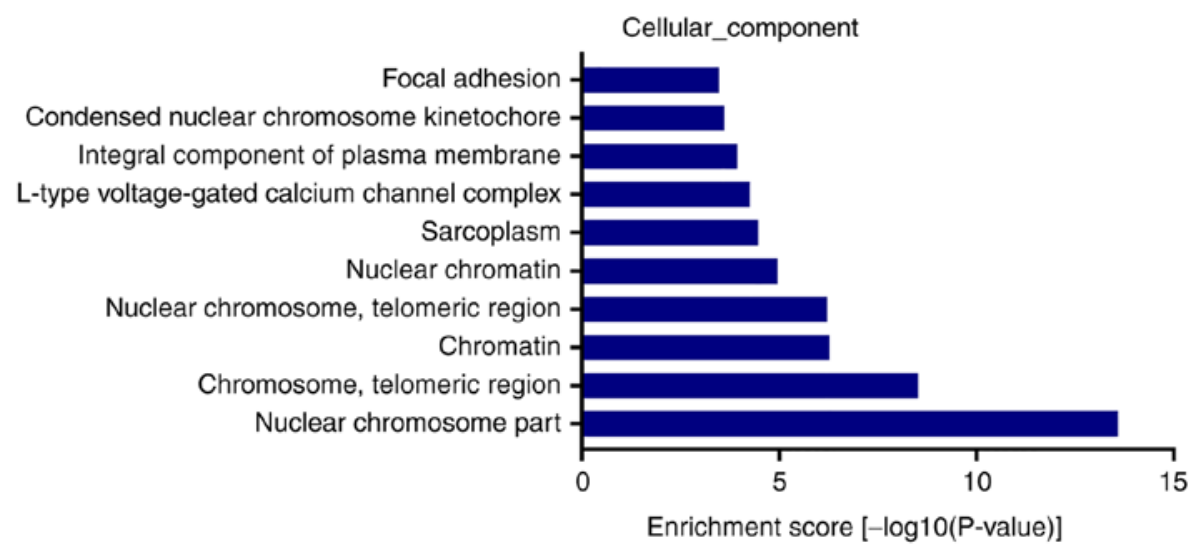

Figure 3. GO analyses for the targeted DE-mRNAs. GO analysis identified (A) biological processes, (B) molecular functions and (C) cellular components. GO, Gene ontology; DE, differentially expressed; mRNA, messenger RNA.

ENST00000398461), and two upregulated mRNAs (CDK1 and CCNB1) and determined their practical variations in the 64 paired non-cancerous and cancer samples from the 64 newly diagnosed BC patients via RT-qPCR (Fig. 5A and Table SIII). The RT-qPCR validation data for aforementioned screened lncRNAs and mRNAs and the sequencing results were relatively consistent (Fig. 5B). For ENST00000598996 and ENST00000524265, the present study also performed RT-qPCR verification in four BC cell lines with different degrees of malignancy and a normal cell line (Fig. 5C).

Prediction of miRNA binding sites, ceRNA and PPI network construction. In the present study, 103 BC-associated lncRNAs were identified that were differentially expressed between $\mathrm{BC}$ tissues vs. paired adjacent non-tumor tissues. Then, the 5 vital miRNAs were predicted though miRanda and DIANA tools (30) (Table III). Target genes that were predicted to bind to these miRNAs were further identified using miRTarBase (31) and TargetScan (32). Eventually, based on the aforementioned analysis, the present study identified an intersection between the mRNAs predicted by lncRNAs and sequencing DE mRNAs. The results presented the 236 intersection mRNAs (Table SII), and certain of them have been affirmed as cancer-associated genes, for instance CDK1, CCNB1, BUB1, EGF, E2F1, E2F2, FEN1, CD36, PARP1 and EFNA1 (33,34).

To date, the functions of most IncRNAs have not been determined. Therefore, the present study constructed a ceRNA and PPI network according to predicted IncRNA-miRNA, miRNA-mRNA and protein-protein degree of interaction, in order to investigate the critical lncRNAs in BC tissues. The 


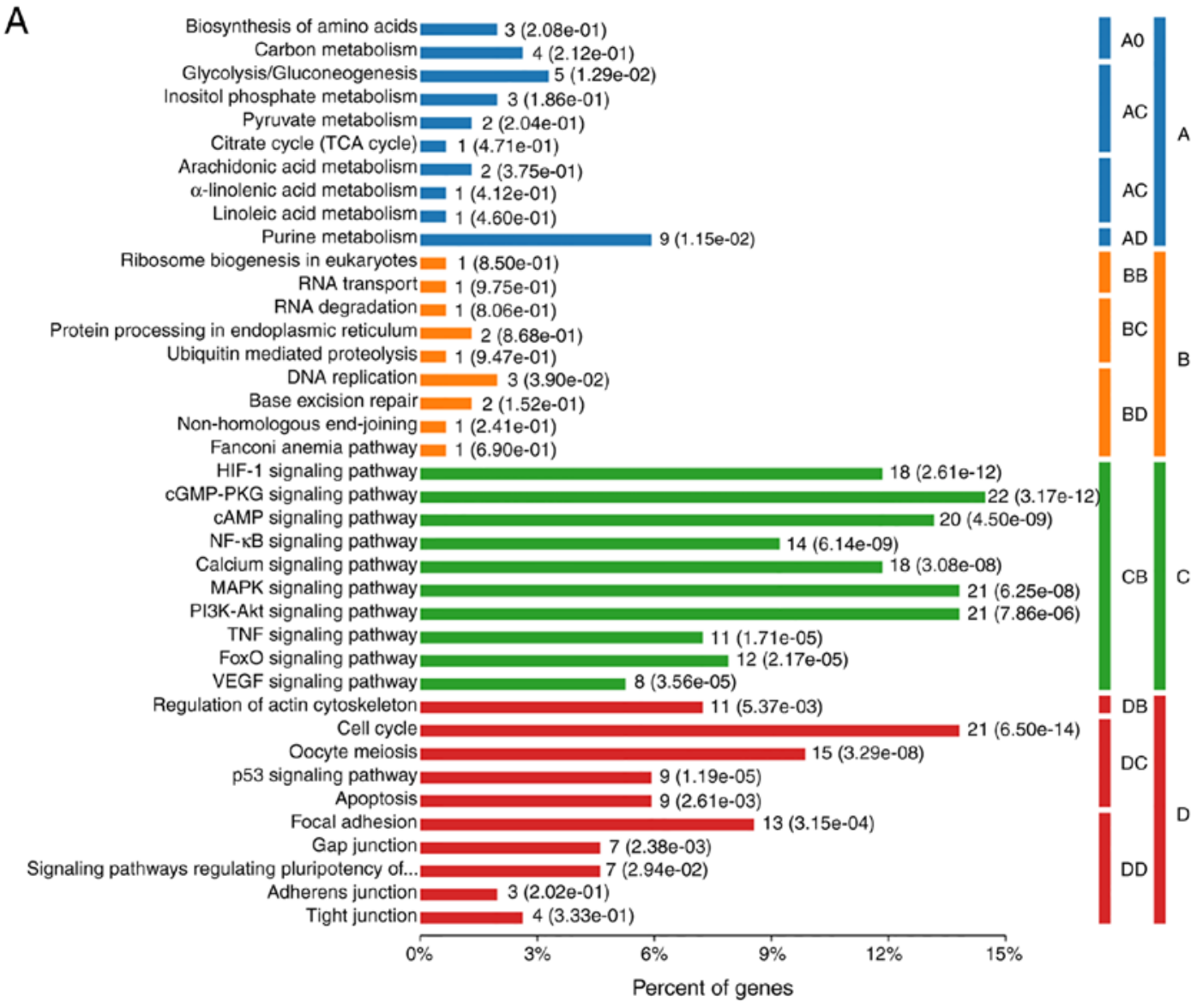

A : Metabolism
B : Genetic information processing
C : Environmental information processing
D : Cellular processes

D : Cellular processes

B

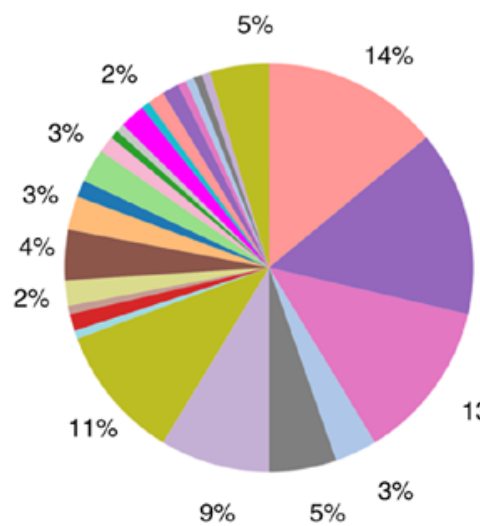

A0 : Global and overview maps

AA : Carbohydrate metabolism

AC : Lipid metabolism

AD : Nucleotide metabolism

BB : Translation

$B C$ : Folding, sorting and degradation

$\mathrm{BD}$ : Replication and repair

CB : Signal transduction

DB : Cell motility

$\mathrm{DC}:$ Cell growth and death

$\mathrm{DD}:$ Cellular community

Unknown

Other KEGG pathways

DNA replication (3.90e-02)

Fc $\gamma$ R-mediated phagocytosis (3.26e-03)

ErbB signaling pathway $(8.58 \mathrm{e}-05)$

FoxO signaling pathway (2.17e-05)

p53 signaling pathway $(1.19 \mathrm{e}-05)$

Platelet activation $(8.29 \mathrm{e}-06)$

PI3K-Akt signaling pathway $(7.86 e-06)$

Cholinergic synapse (4.60e-07)

Renin secretion (6.82e-08)

MAPK signaling pathway $(6.25 \mathrm{e}-08)$

Cacium signaling pathway $(3.08 \mathrm{e}-08)$

Viral carcinogenesis (7.55e-09)

NF-KB signaling pathway $(6.14 \mathrm{e}-09)$

Transcriptional misregulation in cancer $(4.99 \mathrm{e}-09)$

cAMP signaling pathway (4.50e-09)

Progesterone-mediated oocyte maturation (1.34e-09)

Oxytocin signaling pathway $(7.06 \mathrm{e}-11)$

Hepatitis B (1.61e-11)

Alcoholism (1.31e-11)

cGMP-PKG signaling pathway $(3.17 \mathrm{e}-12)$

HIF-1 signaling pathway $(2.61 \mathrm{e}-12)$

AGE-RAGE signaling pathway in diabetic...(1.84e-12;

Pathways in cancer (7.53e-13)

HTLV-I infection (3.84e-13)

Cell cycle $(6.50 \mathrm{e}-14)$

Figure 4. KEGG pathway analyses for the targeted DE-mRNAs. (A) The relevant significantly enriched pathways were determined for these DE-mRNAs. Pathways are presented based on the following sub-categories: Metabolism, Genetic Information Processing, Environmental Information Processing and Cellular Processes. (B) A pie chart is divided into 'slices' representing the percentages corresponding to the KEGG categories revealed. KEGG, Kyoto Encyclopedia of Genes and Genomes; DE, differentially expressed; mRNA, messenger RNA. 

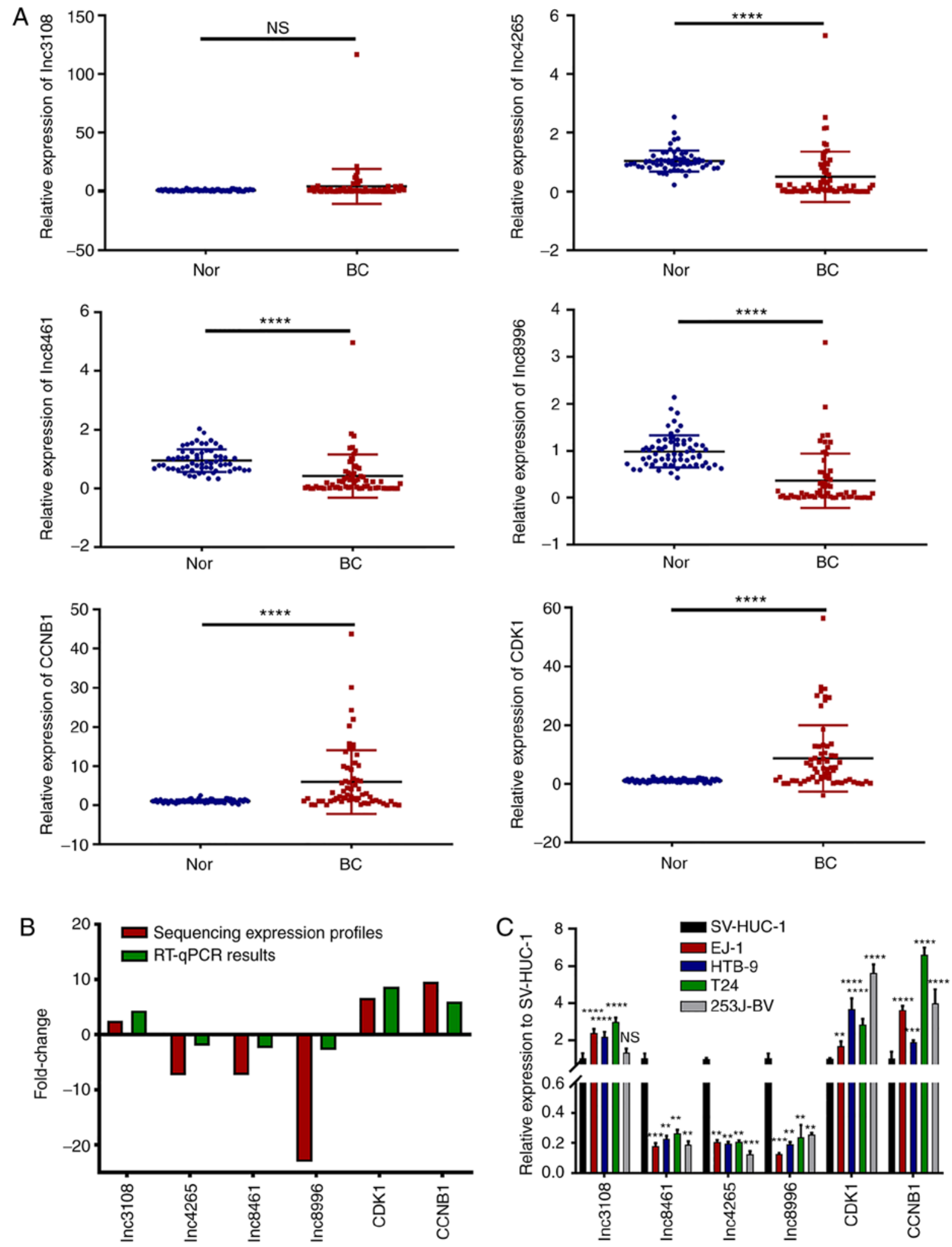

Figure 5. Verification of candidate four lncRNAs and two mRNAs by RT-qPCR (A) Relative expression levels in 64 pairs of BC tissues and non-cancerous tissues. (B) Comparison of fold change $\left(2^{-\Delta \Delta C q}\right)$ of these lncRNAs and mRNAs between the sequencing expression profiles ( $n=5$ paired tissues) and RT-qPCR results ( $\mathrm{n}=64$ paired tissues). (C) Determination of the expression of these selected lncRNAs and mRNAs in four malignant BC cell lines compared with normal urothelial cell line SV-HUC-1. The results are presented as the mean $\pm \mathrm{SD},{ }^{* *} \mathrm{P}<0.01,{ }^{* * *} \mathrm{P}<0.001$ and ${ }^{* * * * *} \mathrm{P}<0.0001$. IncRNA, long non-coding RNA; mRNA, messenger RNA; BC, bladder cancer; RT-qPCR, reverse transcription-quantitative PCR; lnc-3108, ENST00000433108; Inc-4265, ENST00000524265; lnc-8461, ENST00000398461; lnc-8996, ENST00000598996; NS, non-significant; Nor, normal.

present study selected all dysregulated lncRNAs and their 236 targeted DE-mRNAs to construct the ceRNA global network (Fig. S1). The miRNAs hsa-miR-93-5p and hsa-miR-17-5p were equally associated with the majority of lncRNAs and target mRNAs in this global map. Similarly, the present study selected four dysregulated lncRNAs (upregulated 
Table III. Five cancer-related key miRNAs identified for each of the selected four DE lncRNAs.

\begin{tabular}{llllll}
\hline IncRNAs & \multicolumn{1}{c}{ MRE1 } & \multicolumn{1}{c}{ MRE2 } & \multicolumn{1}{c}{ MRE3 } & \multicolumn{1}{c}{ MRE4 } & MRE5 \\
\hline lnc-3108 & hsa-miR-1827 & hsa-miR-1253 & hsa-miR-558 & hsa-miR-1276 & hsa-miR-661 \\
lnc-8996 & hsa-miR-331-3p & hsa-miR-346 & hsa-miR-1299 & hsa-miR-384 & hsa-miR-1231 \\
lnc-4265 & hsa-miR-578 & hsa-miR-602 & hsa-miR-92a-2-5p & hsa-miR-23b-5p & hsa-miR-5088-3p \\
lnc-8461 & hsa-miR-198 & hsa-miR-326 & hsa-miR-146b-3p & hsa-miR-421 & hsa-miR-1294 \\
\hline
\end{tabular}

miRNA, microRNA; DE, differentially expressed; MRE, microRNA response element; lnc-3108, ENST00000433108; lnc-8996, ENST00000598996; lnc-4265, ENST00000524265; lnc-8461, ENST00000398461.

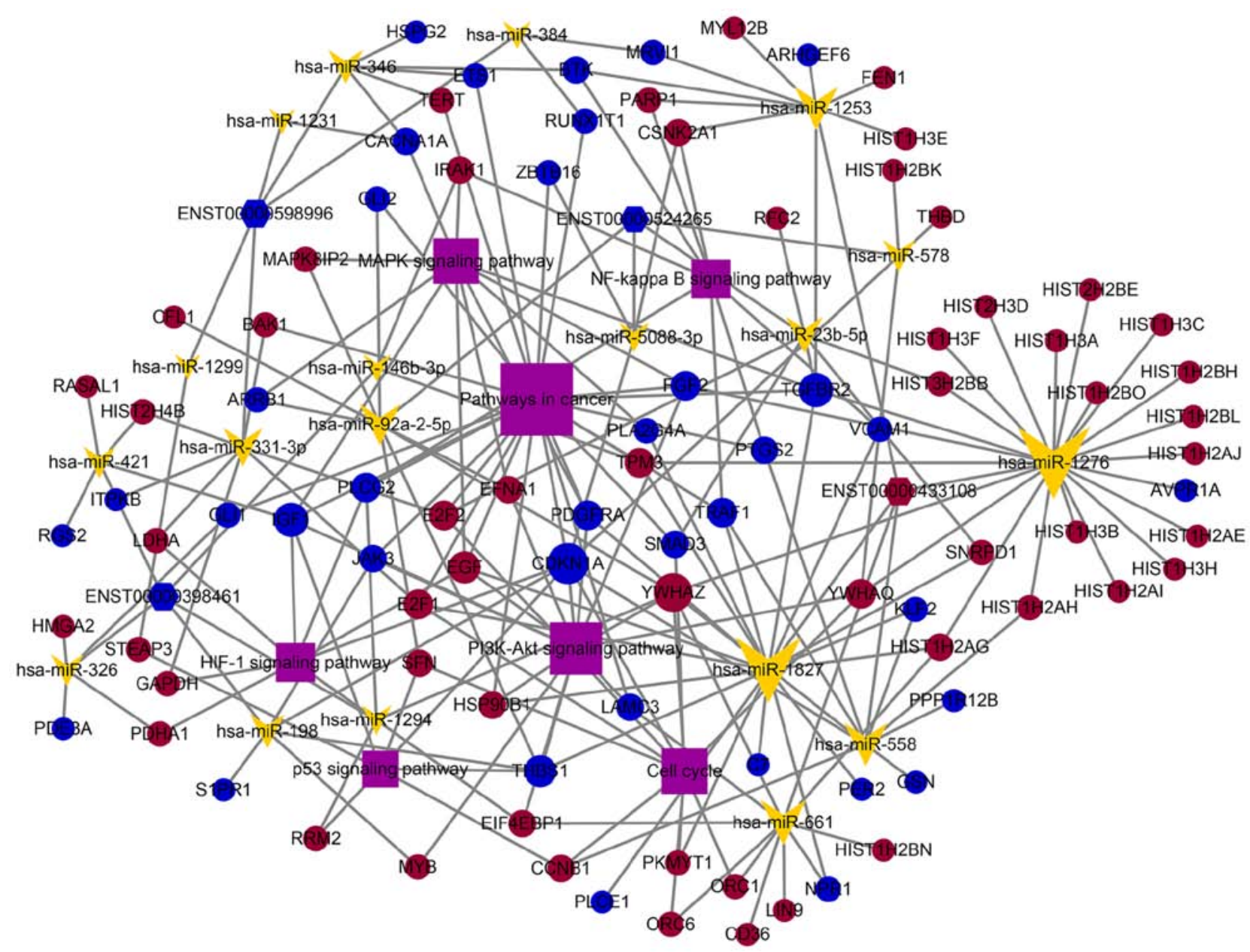

Figure 6. A magnified partial ceRNA network for the selected four lncRNAs was constructed using Cytoscape 3.6 .1 tool. There were 4 lncRNAs, 19 miRNAs, 93 mRNAs and 143 edges in this network. The yellow arrowheads and purple rectangles indicate miRNAs and pathways, respectively. The circles represent mRNAs, hexagons represent lncRNAs, red indicates upregulation, and blue indicates downregulation. ceRNA, competing endogenous RNA; lncRNA, long non-coding RNA; miRNA, microRNA; mRNA, messenger RNA.

ENST00000433108; downregulated ENST00000598996, ENST00000524265 and ENST00000398461) and 93 targeted DE-mRNAs to build the ceRNA local-area network (Fig. 6). The miRNA hsa-miR-1276 was associated with the most lncRNAs and target mRNAs in this localized network. In the ceRNA network map, the preset study demonstrated the top 5 cancer-associated miRNAs that potentially bind to the lncRNAs and the five most likely target genes of each miRNA. Among the 93 intersection genes, the present study also built a PPI network based on the diverse modes of action (Fig. 7 and
Data S1). The ceRNA and PPI networks were established using Cytoscape 3.6.1 and String pool, respectively. Although it provided us with a clear direction in the progression of $\mathrm{BC}$, the deeper functional mechanism of screened DE-lncRNAs and their target genes involved in ceRNA and PPI networks still require further research in the $\mathrm{BC}$ cell lines.

Clinical impact of selected lncRNAs in patients with BC. According to the validation results, ENST00000598996 and ENST00000524265 were the most significantly decreased 


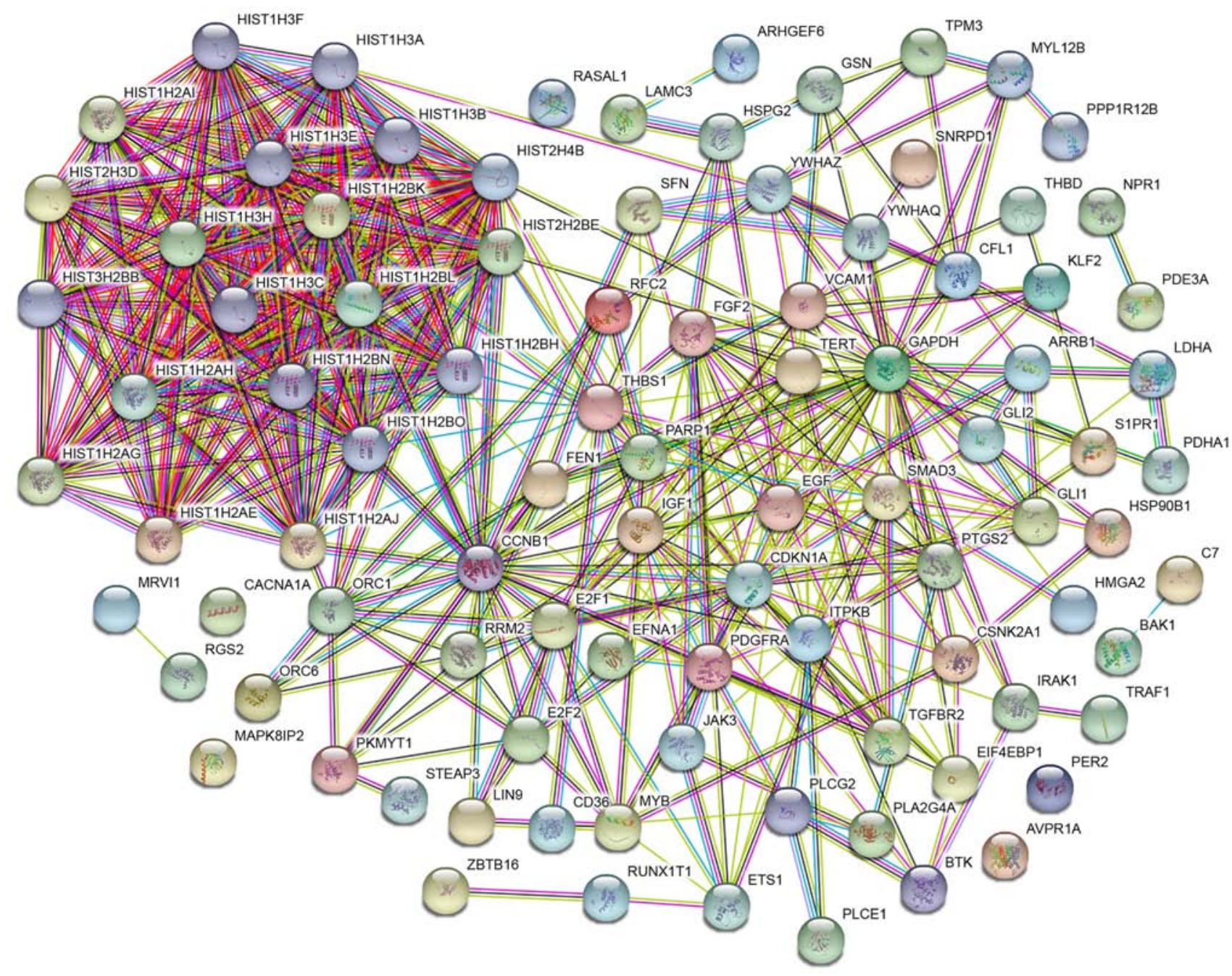

Figure 7. The PPI analysis for 93 target genes were constructed with the String tool. Spheres represent the genes, and lines represent the protein interaction between the genes. Colored nodes represent query proteins and first shell of interactors while white nodes second shell of interactors. The information inside the sphere describes the protein structure: Empty nodes, protein of unknown 3D structure; filled nodes, some 3D structure is known or predicted. Blue-green lines represent known interactions from curated databases; purple lines, known interactions from experimentally determined; green lines, predicted interactions form the gene neighborhood; red lines, predicted interactions from gene fusions; dark blue lines, predicted interactions from gene co-occurrence; yellow lines, interactions from textmining; black lines, interactions from co-expression; light blue lines, interactions form protein homology. PPI, protein-protein interaction.

in $\mathrm{BC}$ specimens compared with those in normal specimens. Subsequently, the present study assessed the correlation between the expression of these four lncRNAs and various clinicopathological characteristics of patients with BC. As presented in Table IV, a decreased expression level of ENST00000598996 and ENST00000524265 was markedly associated with histological stage, tumor pathological stage and lymphatic metastasis, rather than the sex and age of the patients. Subsequently, the ROC curve analysis was conducted in order to estimate the diagnostic performance of these two lncRNAs in patients with BC. The AUC was 0.867 [95\% confidence interval (CI): 0.795-0.939; $\mathrm{P}<0.0001]$ for ENST00000598996, 0.815 (95\% CI: 0.732-0.899, P<0.0001) for ENST00000524265 (Fig. 8A).

In addition, the present study investigated the prognostic value of ENST00000598996 and ENST00000524265 expression in BC by performing a Kaplan-Meier survival analysis of OS and PFS. The results indicated that patients with a lower expression level of ENST00000598996 and
ENST00000524265 had shorter postoperative OS and PFS time than patients with higher expression of ENST00000598996 and ENST00000524265 (Fig. 8B-E). Furthermore, univariate and multivariate Cox regression analyses revealed that ENST00000598996 and ENST00000524265 expression levels were potential prognostic predictors for OS and PFS of patients with BC (Table V).

These results demonstrated an association between downregulation of ENST00000598996 and ENST00000524265 and aggressive tumor characteristics of BC, and may serve as tumor suppressor genes in BC. These results indicated the potential diagnostic and prognostic value of these two lncRNAs in patients with BC.

Both ENST00000598996 and ENST00000524265 play a tumor-suppressive role in BC cells. Next, the biological roles of ENST00000598996 (named lnc8996) and ENST00000524265 (lnc4265) was investigated in BC cells. To achieve this, the BC cell lines EJ-1 and HTB-9 cells were 
Table IV. Correlation analysis between the expression of selected four lncRNAs and clinicopathological characteristics in 64 BC patients.

\begin{tabular}{|c|c|c|c|c|c|c|c|c|c|c|c|c|}
\hline \multirow[b]{2}{*}{$\begin{array}{l}\text { Variables } \\
\text { (total) }\end{array}$} & \multicolumn{2}{|c|}{$\begin{array}{l}\text { lnc-8996 } \\
\text { expression }\end{array}$} & \multirow[b]{2}{*}{ P-value } & \multicolumn{2}{|c|}{$\begin{array}{l}\operatorname{lnc}-4265 \\
\text { expression }\end{array}$} & \multirow[b]{2}{*}{$\mathrm{P}$-value } & \multicolumn{2}{|c|}{$\begin{array}{l}\text { lnc-3108 } \\
\text { expression }\end{array}$} & \multirow[b]{2}{*}{ P-value } & \multicolumn{2}{|c|}{$\begin{array}{c}\text { lnc-8461 } \\
\text { expression }\end{array}$} & \multirow[b]{2}{*}{ P-value } \\
\hline & $\begin{array}{l}\text { Low } \\
n=56\end{array}$ & $\begin{array}{c}\text { High } \\
\mathrm{n}=8\end{array}$ & & $\begin{array}{l}\text { Low } \\
n=53\end{array}$ & $\begin{array}{l}\text { High } \\
n=11\end{array}$ & & $\begin{array}{l}\text { Low } \\
n=32\end{array}$ & $\begin{array}{l}\text { High } \\
n=32\end{array}$ & & $\begin{array}{l}\text { Low } \\
\mathrm{n}=55\end{array}$ & $\begin{array}{l}\text { High } \\
\mathrm{n}=9\end{array}$ & \\
\hline \multicolumn{13}{|l|}{ Sex } \\
\hline Female (7) & 7 & 0 & 0.582 & 5 & 2 & 0.594 & 2 & 5 & 0.426 & 5 & 2 & 0.253 \\
\hline Male (57) & 49 & 8 & & 48 & 9 & & 30 & 27 & & 50 & 7 & \\
\hline \multicolumn{13}{|l|}{ Age } \\
\hline$<65(23)$ & 20 & 3 & $>0.9$ & 17 & 6 & 0.182 & 10 & 13 & 0.603 & 19 & 4 & 0.711 \\
\hline$\geq 65(41)$ & 36 & 5 & & 36 & 5 & & 22 & 19 & & 36 & 5 & \\
\hline \multicolumn{13}{|l|}{ Tumor size ${ }^{a}$} \\
\hline$<3 \mathrm{~cm}(32)$ & 26 & 6 & 0.257 & 27 & 5 & $>0.9$ & 19 & 13 & 0.211 & 26 & 6 & 0.474 \\
\hline$\geq 3 \mathrm{~cm}(32)$ & 30 & 2 & & 26 & 6 & & 13 & 19 & & 29 & 3 & \\
\hline \multicolumn{13}{|l|}{ Multiplicity } \\
\hline Soli. (31) & 26 & 5 & 0.468 & 24 & 7 & 0.331 & 19 & 12 & 0.133 & 27 & 4 & $>0.9$ \\
\hline Mult. (33) & 30 & 3 & & 29 & 4 & & 13 & 20 & & 28 & 5 & \\
\hline \multicolumn{13}{|l|}{$\begin{array}{l}\text { Pathological } \\
\text { stage }\end{array}$} \\
\hline $\mathrm{T} 1-\mathrm{T} 2(36)$ & 29 & 7 & 0.07 & 26 & 10 & $0.017^{\mathrm{b}}$ & 19 & 17 & 0.801 & 28 & 8 & 0.066 \\
\hline T3-T4 (28) & 27 & 1 & & 27 & 1 & & 13 & 15 & & 27 & 1 & \\
\hline \multicolumn{13}{|l|}{$\begin{array}{l}\text { Histologic } \\
\text { grade }\end{array}$} \\
\hline Low (25) & 19 & 6 & $0.048^{b}$ & 17 & 8 & $0.018^{\mathrm{b}}$ & 13 & 12 & $>0.9$ & 18 & 7 & $0.022^{\mathrm{b}}$ \\
\hline High (39) & 37 & 2 & & 36 & 3 & & 19 & 20 & & 37 & 2 & \\
\hline \multicolumn{13}{|l|}{$\begin{array}{l}\text { Lymphatic } \\
\text { metastasis }\end{array}$} \\
\hline No (38) & 30 & 8 & $0.017^{c}$ & 27 & 11 & $0.002^{\mathrm{c}}$ & 19 & 19 & $>0.9$ & 29 & 9 & $0.008^{c}$ \\
\hline Yes (26) & 26 & 0 & & 26 & 0 & & 13 & 13 & & 26 & 0 & \\
\hline
\end{tabular}

${ }^{a}$ The patients were divided into two groups according to the long diameter of the tumor in the median that is $3 \mathrm{~cm}$. ${ }^{\mathrm{b}} \mathrm{P}<0.05$ and ${ }^{\mathrm{c}} \mathrm{P}<0.01 ; \mathrm{Fisher}$ 's exact test. Soli., solitary; Mult., multiple..

transfected with lnc-8996 or lnc-4265 overexpressed plasmid with PCDNA3.1 frame or negative control (NC), respectively. After $48 \mathrm{~h}$ of treatment, the expression levels of lnc-8996 and lnc-4265 were significantly increased compared to NC (Fig. 9A). As presented in Fig. 9B, the proliferation curves detected by CCK- 8 experiments demonstrated that cell growth ability was attenuated followed by upregulation of lnc-8996 or lnc-4265 compared with the vector group (NC) in EJ-1 and HTB-9 cells. The present study demonstrated that enhanced expression of 1nc-8996 or lnc-4265 weakened the clonogenic capacity of EJ-1 and HTB-9 cells using a colony formation assay (Fig. 9C). To research other biological behaviors of lnc-8996 and lnc-4265 in BC, migration and invasion, scratch wound assays and Transwell assays were carried out in the EJ-1 and HTB-9 cells. Both lnc-8996 and lnc-4265 significantly inhibited the wound healing ability of BC cells (Fig. 9D). Furthermore, as presented in Fig. 9E, both migration potential and invasive ability of $\mathrm{BC}$ cells was significantly impaired by lnc-8996 and lnc-4265 overexpression. Collectively, these data clearly revealed that lnc-8996 or lnc-4265 overexpression strongly inhibited the proliferation, migration and invasion of $\mathrm{BC}$ cells in vitro.

\section{Discussion}

Recently, an increasing number of studies have confirmed that lncRNAs are involved in the molecular biological processes including pathogenesis, invasion, metastasis and survival prognosis, of a number of different types of cancer $(16,25,35)$. However, information regarding advanced BC and lncRNAs remains limited.

Hence, the present study performed high-throughput total transcriptome sequencing to identify DE lncRNAs and mRNAs between five matched BC tissues and adjacent normal tissues. Then, the present study obtained 37,684 lncRNAs and 20,266 mRNAs from the two groups. Among them, compared with controls, the present study detected 35 lncRNAs that were significantly upregulated and 68 that 
A

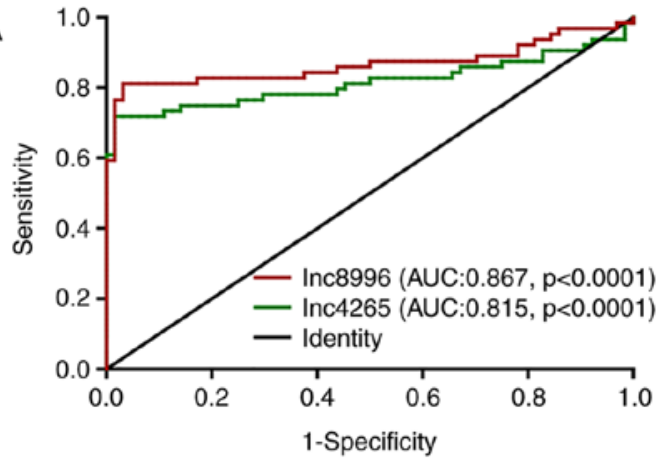

C

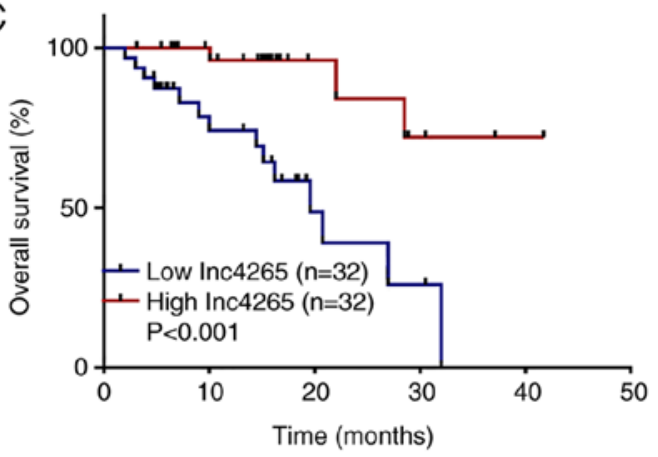

B

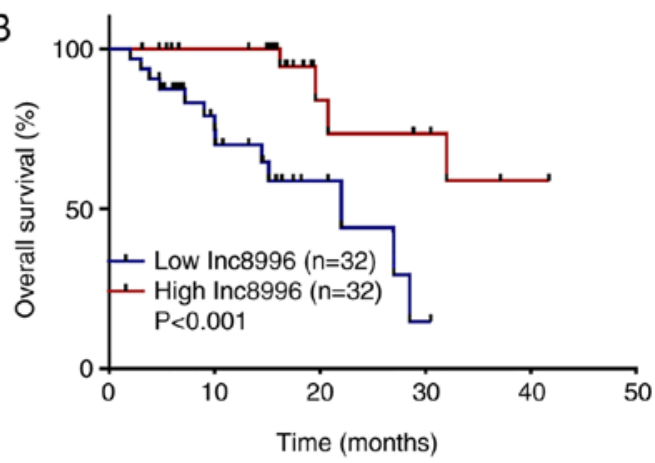

D

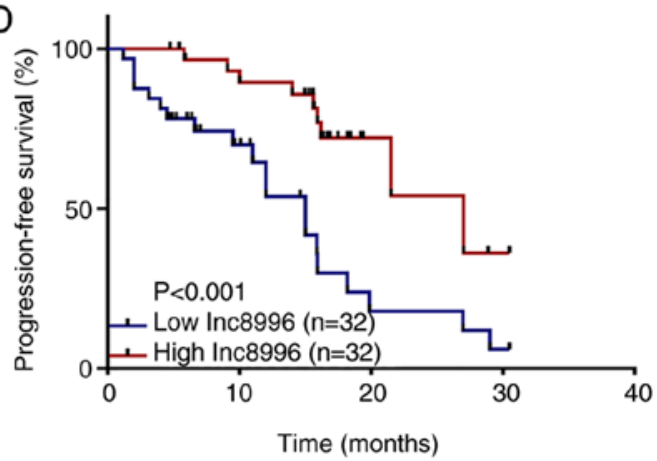

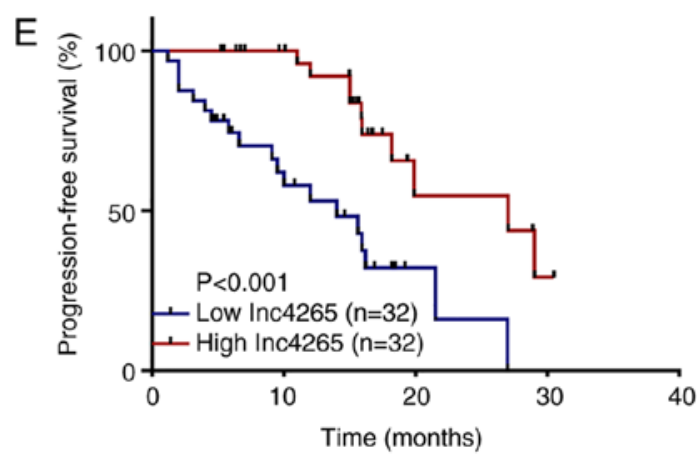

Figure 8. Clinical significance of lnc8996 and lnc4265 in patients with BC. (A) ROC curves of lnc8996 and lnc 4265 differentiating BC samples from adjacent normal samples $(\mathrm{P}<0.0001)$. (B and C) Kaplan-Meier curves for OS based on 1 nc8996 and lnc4265 expression in a group of 64 patients with BC $(\mathrm{P}<0.001)$. Horizontal axis, OS time (months); vertical axis, survival function. (D and E) Kaplan-Meier curves for PFS based on lnc8996 and lnc4265 expression in a group of 64 patients with BC $(\mathrm{P}<0.001)$. Horizontal axis, PFS time (months); vertical axis, survival function. lnc-8996, ENST00000598996; lnc-4265, ENST00000524265; BC, bladder cancer; ROC, receiver operating characteristic; AUC, area under the curve; OS, overall survival; PFS, progression-free survival.

were significantly downregulated in the $\mathrm{BC}$ specimens. To date, a considerable proportion of these DE-lncRNAs have been reported in multiple carcinomas. For example, lncRNA XIST (ENST00000429829) (36,37), TINCR (ENST00000448587) (38,39), MEG3 (ENST00000398460, ENST00000398461 andENST00000452120)(40,41),FENDRR (ENST00000593604 and ENST00000598996) $(42,43)$, and ADAMTS9-AS2 (ENST00000460833, ENST00000474768 and ENST00000481312) $(44,45)$ were observed in gastric, lung and breast cancers; LINC01419 (ENST00000522365) in esophageal (46) and ovarian (47) carcinomas; JHDM1D-AS1(ENST00000566699) in pancreatic cancer (48); MIR205HG (ENST00000433108) in cervical cancer (49); MBNL1-AS1 (ENST00000445466) (50) and PART1 (ENST00000515734) (51) in non-small cell lung cancer. MAGI2-AS3 (ENST00000452320) was observed in hepatocellular carcinoma (52). Notably, it has been reported that
lncRNA XIST (53), MEG3 (54), MAGI2-AS3 (55), FENDRR and ADAMTS9-AS2 (56) act as prognosis markers of patients with $\mathrm{BC}$ or exert significant roles in $\mathrm{BC}$ progression through different cancer-associated pathways. For instance, Kouhsar et al (56) revealed that based on the analysis of the ceRNA networks in the early stage of BC, the IncRNAs FENDRR, ADAMTS9-AS2 and CARMN may regulate MEG3 in NMIBC via harboring some critical miRNAs such as miR-143-3p, miR-34a-3p and miR-106a-5p.

In order to identify the potential functional effects of these dysregulated IncRNAs in BC, the 236 DEmRNAs revealed in the global ceRNA network were used for the GO function and KEGG pathway analyses. As revealed, the significant GO items of these dysregulated genes were 'gene regulation of apoptotic process' (GO:0042981), 'regulation of cell cycle' (GO:0051726), 'positive regulation of gene expression' (GO:0010628) and 'positive regulation of cell 
proliferation' (GO:0008284) in BP. Furthermore, the present study revealed that several cancer-associated pathways were significantly enriched, including 'pathways in cancer', 'cell cycle', 'apoptosis', 'MAPK signaling pathway', 'Focal adhesion', 'PI3K-Akt signaling pathway', 'transcriptional misregulation in cancer', 'cAMP signaling pathway', 'p53 signaling pathway' and 'NF- $\mathrm{BB}$ signaling pathway'. Hence, it is reasonable to assume that these dysregulated lncRNAs in $\mathrm{BC}$ act principally by modulating the cell cycle, cell proliferation and the aforementioned pivotal pathways, which then impact cancer cell aggression. Zhang et al revealed that when compared with paired non-cancerous liver tissues, LINC01419 was significantly upregulated in HCC and may be associated with initiation of HCC via regulating cell cycle genes (57). Wang et al revealed that EPIC1 regulated the cell cycle and promoted breast cancer proliferation (13), and Su et al reported that BLACAT1 affected colorectal cancer cell proliferation by silencing p15 (15). It has also been revealed that downregulation of lncRNA ADAMTS9-AS2 contributed to gastric cancer development through activation of the PI3K/Akt pathway (44). However, although numerous miRNAs, genes and pathways indicated in this ceRNA network have been reported in types of cancer, the detailed molecular mechanism involved in BC development and progression requires further investigation.

Notably, according to their nearby genes, the present study was also able to identify a few tumor-associated lncRNAs altered in BC. ENST00000562848, ENST00000587187, ENST00000398878,ENST00000421206,ENST00000443576 and ENST00000562393 are spliced from the neighboring areas of HOXC6 (58), UHRF1 (59), SULT1A3 (60), GRK5 (61), STARD13 (62) and BDKRB2 (63), which plays a critical role in malignant proliferation, migration and metastasis. Overall, these findings indicated that these lncRNAs are associated with a diverse range of different types of cancer, thereby emphasizing the accuracy of the sequencing data in the present study, and indicating the potential critical role of these lncRNAs in the development of BC as well. Therefore, efforts should be made to ascertain their biological function in BC. These four lncRNAs (upregulated, lnc-3108; downregulated, lnc-8996, lnc-4265 and lnc-8461) were selected and then investigated in another 64 newly diagnosed patients with BC and two lncRNAs (downregulated, lnc-8996 and lnc-4265) in four BC cells by RT-qPCR. These verification results were nearly accordant with the RNA-seq results.

An increasing amount evidence has demonstrated a correlation between IncRNA expression and the clinicopathological characteristics of different types of cancer, such as World Health Organization grade and KPS score in glioma (64), lymph node metastasis and Tumor-Node-Metastasis classification in HCC (65), and invasion depth, tumor stage and lymphatic metastasis in gastric carcinoma (43). In the present study, according to aforementioned validation data, lnc-8996 and lnc-4265 were found to be expressed at significantly lower levels in $\mathrm{BC}$ tissues than in the normal controls, and associated with advanced $\mathrm{T}$ stage, histological grade or lymph node metastasis in patients with BC. Furthermore, overexpression of lnc-8996 and lnc-4265 suppressed BC cell line proliferation, migration and invasion as demonstrated by the in vitro experiments. For lnc-8996, similar results have also been demonstrated in lung adenocarcinoma $(43,66)$. 
A
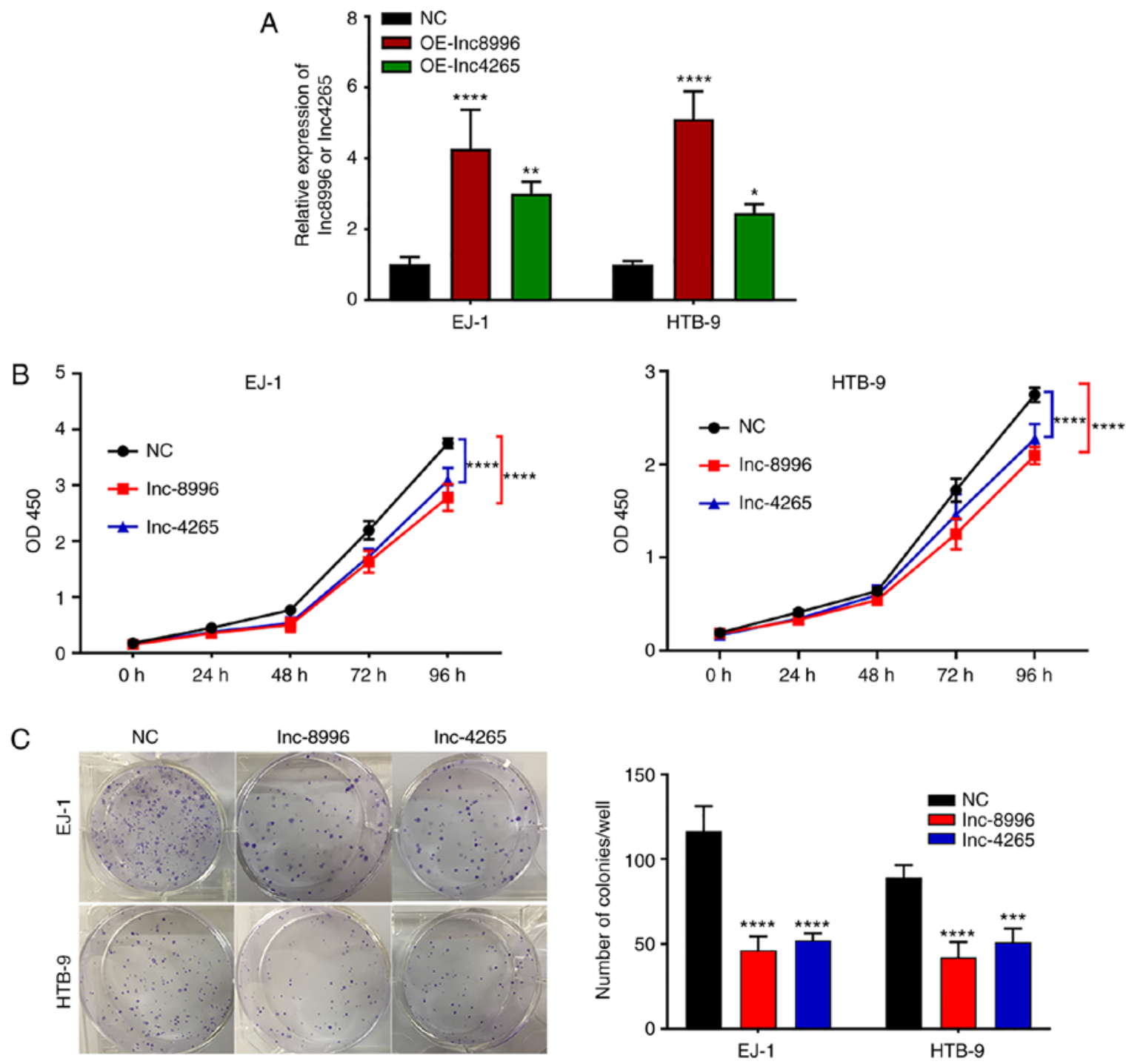

Figure 9. Overexpression of 1 nc 8996 and lnc 4265 weakens the malignant biological behaviors of BC cells. (A) BC cell lines (EJ-1 and HTB-9) were transfected with plasmids and the expression levels of $1 \mathrm{nc} 8996$ and $\operatorname{lnc} 4265$ were significantly increased as revealed by RT-qPCR. (B) CCK-8 (n=5) and (C) colony-formation $(n=3)$ assays revealed that ectopic lnc8996 and lnc4265 expression inhibited EJ-1 and HTB-9 cell proliferation. Bars represent the number of colonies.

Notably, ectopic expression of lnc-8996 and lnc-4265 affected the migration and invasion abilities of BC cells to a greater extent than the proliferation ability. A potential complex biological regulatory mechanism of $1 \mathrm{nc}-8996$ or lnc-4265 may be mainly responsible for the discrepancies. With regard to this phenomenon, similar results were also revealed in numerous studies $(67,68)$.

Furthermore, prior studies suggested that lncRNAs function as diagnostic or prognostic biomarkers and therapeutic targets for malignancies, such as LINC00261 and lncRNA FOXD2-AS1 for gastric cancer $(18,35)$ and ZEB1-AS1 for hepatocellular carcinoma $(8,16)$. In accordance with the results of these previous studies, by applying Kaplan-Meier analysis and log-rank tests, the present study indicated that patients with BC with low lnc-8996 or lnc-4265 expression levels had poorer OS and PFS compared with those patients with higher levels. Furthermore, after the univariate and multivariate Cox regression analyses, lnc-8996 and lnc-4265 could serve as potential prognostic markers for both OS and PFS. In the multivariate Cox regression analysis, only lnc-8996 and lnc-4265, rather than $\mathrm{T}$ stage and lymph metastasis were statistically significant. The most likely reason for this result was a multicollinearity problem. With regard to this phenomenon, similar results were also found in numerous tumor studies (69-71). Next, the prediction power of each of the two lncRNAs was estimated on the basis of the ROC curve, and the results revealed that lnc-8996 and lnc- 4265 had better diagnostic prediction power (AUC equals 0.867 and 0.815 , respectively). These results indicated that decreased expression of lnc-8996 and lnc-4265 may act as significant biomarkers for the diagnosis and prognosis of $\mathrm{BC}$.

LncRNAs have been reported to exert multifaceted roles by functioning as cytoplasmic miRNA sponges, combined with RNA-binding proteins or other functional proteins, and acting as nuclear transcriptional regulators $(13,17,72)$. Among these roles, functioning as an miRNA sponge represents the most classic function, which has been confirmed in various diseases, such as bladder carcinoma (23), colorectal cancer (14) and lung cancer (37). Accordingly, by employing bioinformatics analysis, the present study first revealed 


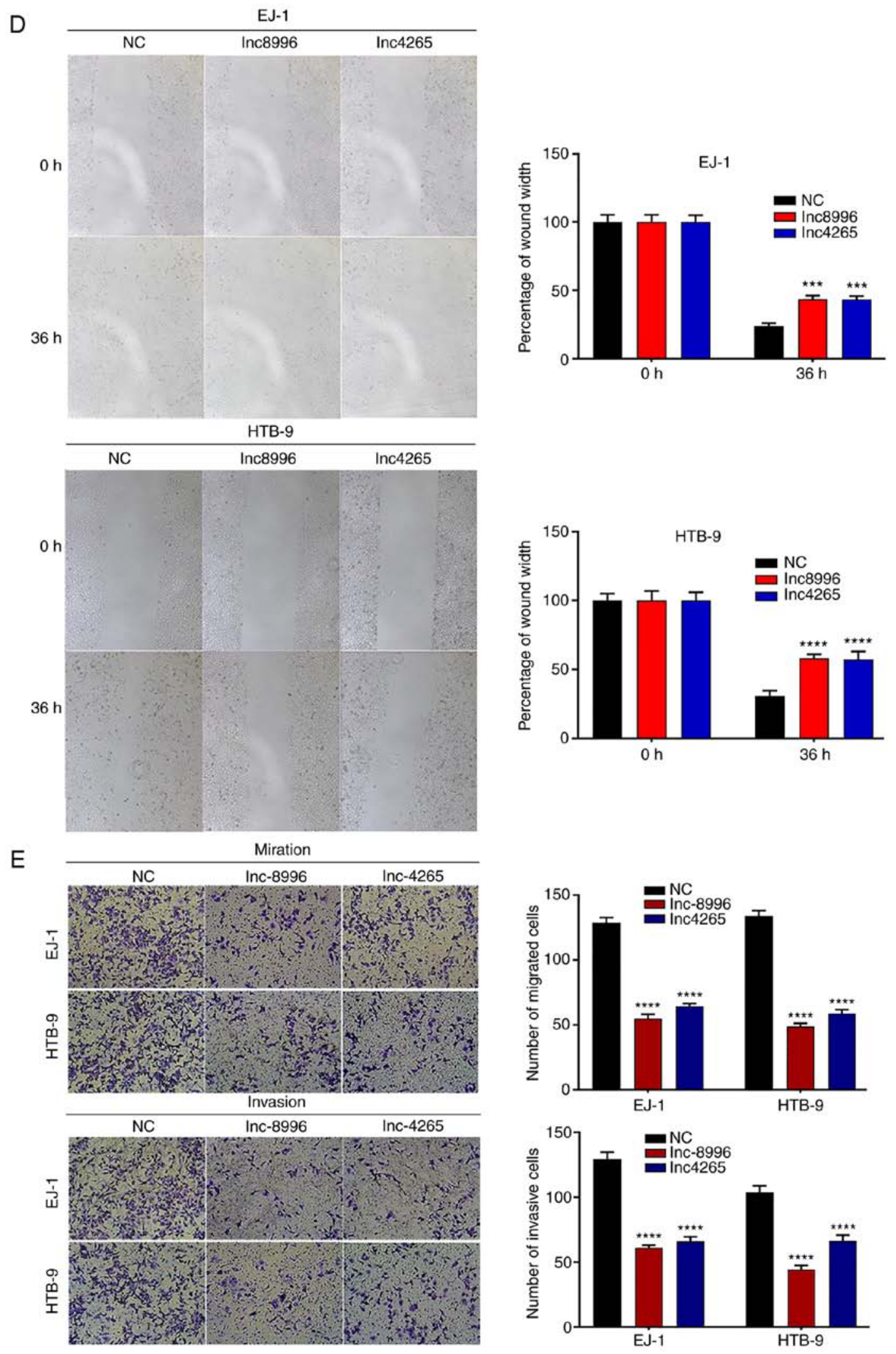

Figure 9. Continued. Overexpression of lnc8996 and lnc4265 weakens the malignant biological behaviors of BC cells. The effect of $1 \mathrm{nc} 8996$ and $1 \mathrm{nc} 4265$ on cell migration and invasion capabilities was evaluated by (D) scratch wound healing, (E) Transwell migration and invasion assays in EJ-1 and HTB-9 cells, respectively. Bars represent (D) the migration rate, and (E) the number of migrated and invasive cells. Scale bar, $50 \mu \mathrm{m}$. Data are presented as the means $\pm \mathrm{SD}$ of three independent experiments; ${ }^{*} \mathrm{P}<0.05,{ }^{* *} \mathrm{P}<0.01,{ }^{* * * *} \mathrm{P}<0.001$ and ${ }^{* * * *} \mathrm{P}<0.0001$. Inc-8996, ENST00000598996; Inc-4265, ENST00000524265; BC, bladder cancer; RT-qPCR, reverse transcription-quantitative PCR; CCK-8, Cell Counting Kit-8; OE, overexpression; NC, negative control.

that these four lncRNAs may interact with 19 types of predicted crucial miRNAs and therefore, the presumptive
93 key DE-genes stemmed from the sequencing results that were used to form ceRNA and PPI networks. Notably, in 
the ceRNA network, it was revealed that miR-661 (73) and miR-326 (74) exhibited high and low expression in cancer tissues, respectively. Li et al also revealed that upregulated miR-558 promoted cell migration, invasion and angiogenesis by targeting HPSE in T24T and UMUC3 cells (75). CCNB1, the $\mathrm{G} 2 / \mathrm{M}$ transition member of the cell cycle, was significantly upregulated in BC tumor tissues, which was consistent with a previous study (76). Notably, these studies also reflect the accuracy of the predicted ceRNA network in the present study.

In conclusion, the present study presented general perspectives of DElncRNAs and DEmRNAs in BC samples vs. adjacent non-BC samples using RNA high-throughput sequencing. Four lncRNAs (lnc-3108, lnc-8996, lnc-4265 and lnc-8461) were further validated in both BC tissues and cell lines via RT-qPCR. The results revealed that lnc-8996 and lnc-4265 could be potential diagnostic biomarkers for BC. It was also revealed that lnc-8996 and lnc-4265 expression levels were markedly downregulated and closely associated with unfavorable clinicopathological parameters and poor survival prognoses. In addition, through bioinformatics, the present study predicted a number of potential gene regulatory networks and signaling pathways associated with these DE-lncRNAs. Ultimately, lnc-8996 and lnc-4265 overexpression retarded bladder cancer cell proliferation, migration and invasion in vitro. All these results indicated that lnc-8996 and lnc-4265 may be potential biomarkers for diagnosis and prognosis, as well as promising therapeutic targets in patients with BC. However, larger clinical samples and deeper insights into the regulatory mechanisms of these lncRNAs are required to confirm the present data.

\section{Acknowledgements}

We would like to thank Cloud-Seq Biotech Co., Ltd. (Shanghai, China) for the high-throughput RNA-Seq service.

\section{Funding}

The present study received financial support from the Key Project of Health Industry in Tianjin (grant no. 16KG119), the Natural Science Foundation Project of Tianjin (grant no. 18PTLCSY00010), the Tianjin Urological Key Laboratory Foundation (grant no. 2017ZDSYS13) and the Youth Fund of Tianjin Medical University Second Hospital (grant no. 2018ydey09).

\section{Availability of data and materials}

The datasets used and/or analyzed during the current study are available from the corresponding author on reasonable request.

\section{Authors' contributions}

$\mathrm{HH}$ and CS conceived and designed the study. DT and ZW collected the clinical specimens. CS, YW, LD and SG performed the experiments and analyzed the results. CS wrote the manuscript. $\mathrm{HH}, \mathrm{LX}, \mathrm{YQ}, \mathrm{YW}$ and $\mathrm{ZZ}$ edited the manuscript and provided critical comments. All authors read and approved the final version of this manuscript and agree to be accountable for all aspects of the research in ensuring that the accuracy or integrity of any part of the work are appropriately investigated and resolved.

\section{Ethics approval and consent to participate}

The protocol of the present study was conducted based on the Declaration of Helsinki and approved by the Medical Review Committee of the Second Hospital of Tianjin Medical University. Written informed consent was obtained from all patients for the use of their tissue in this study.

\section{Patient consent for publication}

Not applicable.

\section{Competing interests}

The authors declare that they have no competing interests.

\section{References}

1. Bray F, Ferlay J, Soerjomataram I, Siegel RL, Torre LA and Jemal A: Global cancer statistics 2018: GLOBOCAN estimates of incidence and mortality worldwide for 36 cancers in 185 countries. CA Cancer J Clin 68: 394-424, 2018.

2. Siegel RL, Miller KD and Jemal A: Cancer statistics, 2019. CA Cancer J Clin 69: 7-34, 2019.

3. Di Meo A, Bartlett J, Cheng Y, Pasic MD and Yousef GM: Liquid biopsy: A step forward towards precision medicine in urologic malignancies. Mol Cancer 16: 80, 2017.

4. Singh R, Ansari JA, Maurya N, Mandhani A, Agrawal V and Garg M: Epithelial-to-mesenchymal transition and its correlation with clinicopathologic features in patients with urothelial carcinoma of the bladder. Clin Genitourin Cancer 15: e187-e197, 2017.

5. Cao W, Zhao Y, Wang L and Huang X: Circ0001429 regulates progression of bladder cancer through binding miR-205-5p and promoting VEGFA expression. Cancer Biomark 25: 101-113, 2019.

6. Li B, Xie F, Zheng FX, Jiang GS, Zeng FQ and Xiao XY: Overexpression of CircRNA BCRC4 regulates cell apoptosis and MicroRNA-101/EZH2 signaling in bladder cancer. J Huazhong Univ Sci Technolog Med Sci 37: 886-890, 2017.

7. Antoni S, Ferlay J, Soerjomataram I, Znaor A, Jemal A and Bray F: Bladder cancer incidence and mortality: A global overview and recent trends. Eur Urol 71: 96-108, 2017.

8. Qiu L, Tang Q, Li G and Chen K: Long non-coding RNAs as biomarkers and therapeutic targets: Recent insights into hepatocellular carcinoma. Life Sci 191: 273-282, 2017.

9. Tang Y, Cheung BB, Atmadibrata B, Marshall GM, Dinger ME, Liu PY and Liu T: The regulatory role of long noncoding RNAs in cancer. Cancer Lett 391: 12-19, 2017.

10. Vishnubalaji R, Shaath H, Elkord E and Alajez NM: Long non-coding RNA (lncRNA) transcriptional landscape in breast cancer identifies LINC01614 as non-favorable prognostic biomarker regulated by TGF $\beta$ and focal adhesion kinase (FAK) signaling. Cell Death Discov 5: 109, 2019.

11. Yang H, Chen T, Xu S, Zhang S and Zhang M: Long noncoding RNA FOXC2-AS1 predicts poor survival in breast cancer patients and promotes cell proliferation. Oncol Res 27: 219-226, 2019.

12. Chen C, He W, Huang J, Wang B, Li H, Cai Q, Su F, Bi J, Liu H, Zhang B, et al: LNMAT1 promotes lymphatic metastasis of bladder cancer via CCL2 dependent macrophage recruitment. Nat Commun 9: 3826, 2018.

13. Wang Z, Yang B, Zhang M, Guo W, Wu Z, Wang Y, Jia L, Li S, Xie W and Yang D; Cancer Genome Atlas Research Network: lncRNA epigenetic landscape analysis identifies EPIC1 as an oncogenic lncRNA that interacts with MYC and promotes cell-cycle progression in cancer. Cancer Cell 33: 706-720, 2018. 
14. Xu M, Chen X, Lin K, Zeng K, Liu X, Pan B, Xu X, Xu T, $\mathrm{Hu} X$, Sun L, et al: The long noncoding RNA SNHG1 regulates colorectal cancer cell growth through interactions with EZH2 and miR-154-5p. Mol Cancer 17: 141, 2018.

15. Su J, Zhang E, Han L, Yin D, Liu Z, He X, Zhang Y, Lin F, Lin Q, Mao P, et al: Long noncoding RNA BLACAT1 indicates a poor prognosis of colorectal cancer and affects cell proliferation by epigenetically silencing of p15. Cell Death Dis 8: e2665, 2017.

16. Jin L, He Y, Tang S and Huang S: LncRNA GHET1 predicts poor prognosis in hepatocellular carcinoma and promotes cell proliferation by silencing KLF2. J Cell Physiol 233: 4726-4734, 2018.

17. Wang Y, Yang L, Chen T, Liu X, Guo Y, Zhu Q, Tong X, Yang W, $\mathrm{Xu}$ Q, Huang D and Tu K: A novel lncRNA MCM3AP-AS1 promotes the growth of hepatocellular carcinoma by targeting miR-194-5p/FOXA1 axis. Mol Cancer 18: 28, 2019.

18. Fan Y, Wang YF, Su HF, Fang N, Zou C, Li WF and Fei ZH: Decreased expression of the long noncoding RNA LINC00261 indicate poor prognosis in gastric cancer and suppress gastric cancer metastasis by affecting the epithelial-mesenchymal transition. J Hematol Oncol 9: 57, 2016.

19. Li X, Xiao X, Chang R and Zhang C: Comprehensive bioinformatics analysis identifies lncRNA HCG22 as a migration inhibitor in esophageal squamous cell carcinoma. J Cell Biochem 121: 468-481, 2020

20. Chen W, Hang Y, Xu W, Wu J, Chen L, Chen J, Mao Y, Song J, Song $\mathrm{J}$ and Wang H: BLACAT1 predicts poor prognosis and serves as oncogenic lncRNA in small-cell lung cancer. J Cell Biochem 120: 2540-2546, 2019.

21. Fang C, He W, Xu T, Dai J, Xu L and Sun F: Upregulation of lncRNA DGCR5 correlates with better prognosis and inhibits bladder cancer progression via transcriptionally facilitating P21 expression. J Cell Physiol 234: 6254-6262, 2019.

22. Shan H, Yang Y, Zhu X, Han X, Zhang $P$ and Zhang $X$ : FAM83H-AS1 is associated with clinical progression and modulates cell proliferation, migration, and invasion in bladder cancer. J Cell Biochem 120: 4687-4693, 2019.

23. Rui X, Wang L, Pan H, Gu T, Shao S and Leng J: LncRNA GAS6-AS2 promotes bladder cancer proliferation and metastasis via GAS6-AS2/miR-298/CDK9 axis. J Cell Mol Med 23. 865-876, 2019.

24. Xu Z, Huang B, Zhang Q, He X, Wei H and Zhang D: NOTCH1 regulates the proliferation and migration of bladder cancer cells by cooperating with long non-coding RNA HCG18 and microRNA-34c-5p. J Cell Biochem 120: 6596-6604, 2019.

25. Cao X, Xu J and Yue D: LncRNA-SNHG16 predicts poor prognosis and promotes tumor proliferation through epigenetically silencing p21 in bladder cancer. Cancer Gene Ther 25: 10-17, 2018.

26. Zhai $\mathrm{X}$ and $\mathrm{Xu} \mathrm{W}$ : Long noncoding RNA ATB promotes proliferation, migration, and invasion in bladder cancer by suppressing MicroRNA-126. Oncol Res 26: 1063-1072, 2018.

27. Tuo Z, Zhang J and Xue W: LncRNA TP73-AS1 predicts the prognosis of bladder cancer patients and functions as a suppressor for bladder cancer by EMT pathway. Biochem Biophys Res Commun 499: 875-881, 2018.

28. Livak KJ and Schmittgen TD: Analysis of relative gene expression data using real-time quantitative PCR and the 2(-Delta Delta C(T)) method. Methods 25: 402-408, 2001.

29. Huang da W, Sherman BT and Lempicki RA: Systematic and integrative analysis of large gene lists using DAVID bioinformatics resources. Nat Protoc 4: 44-57, 2009.

30. Paraskevopoulou MD, Vlachos IS, Karagkouni D, Georgakilas G, Kanellos I, Vergoulis T, Zaggana K, Tsanakas P, Floros E, Dalamagas $\mathrm{T}$ and Hatzigeorgiou AG: DIANA-LncBase v2: Indexing microRNA targets on non-coding transcripts. Nucleic Acids Res 44: D231-D238, 2016.

31. Chou CH, Shrestha S, Yang CD, Chang NW, Lin YL, Liao KW, Huang WC, Sun TH, Tu SJ, Lee WH, et al: MiRtarBase update 2018: A resource for experimentally validated microRNA-target interactions. Nucleic Acids Res 46: D296-D302, 2018.

32. Agarwal V, Bell GW, Nam JW and Bartel DP: Predicting effective microRNA target sites in mammalian mRNAs. Elife 4 e05005, 2015.

33. Zhuang L, Yang Z and Meng Z: Upregulation of BUB1B CCNB1, CDC7, CDC20, and MCM3 in tumor tissues predicted worse overall survival and disease-free survival in hepatocellular carcinoma patients. Biomed Res Int 2018: 7897346, 2018.

34. Yao H, Lu F and Shao Y: The E2F family as potential biomarkers and therapeutic targets in colon cancer. Peer J 8: e8562, 2020.
35. Xu TP, Wang WY, Ma P, Shuai Y, Zhao K, Wang YF, Li W, Xia R, Chen WM, Zhang EB and Shu YQ: Upregulation of the long noncoding RNA FOXD2-AS1 promotes carcinogenesis by epigenetically silencing EphB3 through EZH2 and LSD1, and predicts poor prognosis in gastric cancer. Oncogene 37: 5020-5036, 2018.

36. Xing F, Liu Y, Wu SY, Wu K, Sharma S, Mo YY, Feng J, Sanders S, Jin G, Singh R, et al: Loss of XIST in breast cancer activates MSN-c-Met and reprograms microglia via exosomal miRNA to promote brain metastasis. Cancer Res 78: 4316-4330, 2018.

37. Li C, Wan L, Liu Z, Xu G, Wang S, Su Z, Zhang Y, Zhang C, Liu X, Lei Z and Zhang HT: Long non-coding RNA XIST promotes TGF- $\beta$-induced epithelial-mesenchymal transition by regulating miR-367/141-ZEB2 axis in non-small-cell lung cancer. Cancer Lett 418: 185-195, 2018.

38. Gao YW, Ma F, Xie YC, Ding MG, Luo LH, Jiang S, Rao L and Liu XL: Sp1-induced upregulation of the long noncoding RNA TINCR inhibits cell migration and invasion by regulating miR-107/miR-1286 in lung adenocarcinoma. Am J Transl Res 11: 4761-4775, 2019.

39. Chen Z, Liu H, Yang H, Gao Y, Zhang G and Hu J: The long noncoding RNA, TINCR, functions as a competing endogenous RNA to regulate PDK1 expression by sponging miR-375 in gastric cancer. Onco Targets Ther 10: 3353-3362, 2017.

40. Terashima M, Tange S, Ishimura A and Suzuki T: MEG3 long noncoding RNA contributes to the epigenetic regulation of epithelial-mesenchymal transition in lung cancer cell lines. J Biol Chem 292: 82-99, 2017.

41. Zhang Y, Wu J, Jing H, Huang G, Sun Z and Xu S: Long noncoding RNA MEG3 inhibits breast cancer growth via upregulating endoplasmic reticulum stress and activating NF- $\kappa \mathrm{B}$ and p53. J Cell Biochem 120: 6789-6797, 2019.

42. Gong F, Dong D, Zhang T and Xu W: Long non-coding RNA FENDRR attenuates the stemness of non-small cell lung cancer cells via decreasing multidrug resistance gene 1 (MDR1) expression through competitively binding with RNA binding protein hur. Eur J Pharmacol 853: 345-352, 2019.

43. Xu TP, Huang MD, Xia R, Liu XX, Sun M, Yin L, Chen WM, Han L, Zhang EB, Kong R, et al: Decreased expression of the long non-coding RNA FENDRR is associated with poor prognosis in gastric cancer and FENDRR regulates gastric cancer cell metastasis by affecting fibronectin 1 expression. J Hematol Oncol 7: 63, 2014.

44. Cao B, Liu C and Yang G: Down-regulation of lncRNA ADAMTS9-AS2 contributes to gastric cancer development via activation of PI3K/Akt pathway. Biomed Pharmacother 107: 185-193, 2018.

45. Deva Magendhra Rao AK, Patel K, Korivi Jyothiraj S, Meenakumari B, Sundersingh S, Sridevi V, Rajkumar T, Pandey A, Chatterjee A, Gowda H and Mani S: Identification of lncRNAs associated with early-stage breast cancer and their prognostic implications. Mol Oncol 13: 1342-1355, 2019.

46. Chen JL, Lin ZX, Qin YS, She YQ, Chen Y, Chen C, Qiu GD, Zheng JT, Chen ZL and Zhang SY: Overexpression of long noncoding RNA LINC01419 in esophageal squamous cell carcinoma and its relation to the sensitivity to 5-fluorouracil by mediating GSTP1 methylation. Ther Adv Med Oncol 11: $1758835919838958,2019$.

47. Chen Y, Bi F, An Y and Yang Q: Identification of pathological grade and prognosis-associated lncRNA for ovarian cancer. J Cell Biochem 120: 14444-14454, 2019.

48. Kondo A, Nonaka A, Shimamura T, Yamamoto S, Yoshida T, Kodama T, Aburatani $\mathrm{H}$ and Osawa T: Long noncoding RNA JHDM1D-AS1 promotes tumor growth by regulating angiogenesis in response to nutrient starvation. Mol Cell Biol 37: e00125-e00117, 2017.

49. Li Y, Wang $\mathrm{H}$ and Huang $\mathrm{H}$ : Long non-coding RNA MIR205HG function as a ceRNA to accelerate tumor growth and progression via sponging miR-122-5p in cervical cancer. Biochem Biophys Res Commun 514: 78-85, 2019

50. Li P, Xing W, Xu J, Yuan D, Liang G, Liu B and Ma H: MicroRNA-301b-3p downregulation underlies a novel inhibitory role of long non-coding RNA MBNL1-AS1 in non-small cell lung cancer. Stem Cell Res Ther 10: 144, 2019.

51. Zhu D, Yu Y, Wang W, Wu K, Liu D, Yang Y, Zhang C, Qi Y and Zhao S: Long noncoding RNA PART1 promotes progression of non-small cell lung cancer cells via JAK-STAT signaling pathway. Cancer Med 8: 6064-6081, 2019. 
52. Yin Z, Ma T, Yan J, Shi N, Zhang C, Lu X, Hou B and Jian Z LncRNA MAGI2-AS3 inhibits hepatocellular carcinoma cell proliferation and migration by targeting the miR-374b-5p/SMG1 signaling pathway. J Cell Physiol 234: 18825-18836, 2019.

53. Hu B, Shi G, Li Q, Li W and Zhou H: Long noncoding RNA XIST participates in bladder cancer by downregulating p53 via binding to TET1. J Cell Biochem 120: 6330-6338, 2019.

54. Shan G, Tang T, Xia Y and Qian HJ: MEG3 interacted with miR-494 to repress bladder cancer progression through targeting PTEN. J Cell Physiol 235: 1120-1128, 2020.

55. Wang F, Zu Y, Zhu S, Yang Y, Huang W, Xie H and Li G: Long noncoding RNA MAGI2-AS3 regulates CCDC19 expression by sponging miR-15b-5p and suppresses bladder cancer progression. Biochem Biophys Res Commun 507: 231-235, 2018.

56. Kouhsar M, Azimzadeh Jamalkandi S, Moeini A and Masoudi-Nejad A: Detection of novel biomarkers for early detection of non-muscle-invasive bladder cancer using competing endogenous RNA network analysis. Sci Rep 9: 8434, 2019.

57. Zhang H, Zhu C, Zhao Y, Li M, Wu L, Yang X, Wan X, Wang A, Zhang MQ, Sang $X$ and Zhao $H$ : Long non-coding RNA expression profiles of hepatitis $\mathrm{C}$ virus-related dysplasia and hepatocellular carcinoma. Oncotarget 6: 43770-43778, 2015.

58. Li PD, Chen P, Peng X, Ma C, Zhang WJ and Dai XF: HOXC6 predicts invasion and poor survival in hepatocellular carcinoma by driving epithelial-mesenchymal transition. Aging (Albany NY) 10: 115-130, 2018.

59. Hu Q, Qin Y, Ji S, Xu W, Liu W, Sun Q, Zhang Z, Liu M, Ni Q, $\mathrm{Yu} \mathrm{X}$ and Xu X: UHRF1 promotes aerobic glycolysis and proliferation via suppression of SIRT4 in pancreatic cancer. Cancer Lett 452: 226-236, 2019.

60. Zou J, Li H, Huang Q, Liu X, Qi X, Wang Y, Lu L and Liu Z: Dopamine-induced SULT1A3/4 promotes EMT and cancer stemness in hepatocellular carcinoma. Tumour Biol 39: 1010428317719272,2017

61. Jiang LP, Fan SQ, Xiong QX, Zhou YC, Yang ZZ, Li GF, Huang YC, Wu MG, Shen QS, Liu K, et al: GRK5 functions as an oncogenic factor in non-small-cell lung cancer. Cell Death Dis 9: 295,2018

62. Chen L, Hu W, Li G, Guo Y, Wan Z and Yu J: Inhibition of miR-9-5p suppresses prostate cancer progress by targeting starD13. Cell Mol Biol Lett 24: 20, 2019.

63. Zhou Y, Wang W, Wei R, Jiang G, Li F, Chen X, Wang X, Long S, $\mathrm{Ma} \mathrm{D}$ and $\mathrm{Xi}$ L: Serum bradykinin levels as a diagnostic marker in cervical cancer with a potential mechanism to promote VEGF expression via BDKRB2. Int J Oncol 55: 131-141, 2019.

64. Chen XD, Zhu MX and Wang SJ: Expression of long non-coding RNA MAGI2-AS3 in human gliomas and its prognostic significance. Eur Rev Med Pharmacol Sci 23 3455-3460, 2019.

65. Ni W, Zhang Y, Zhan Z, Ye F, Liang Y, Huang J, Chen K, Chen L and Ding Y: A novel lncRNA uc.134 represses hepatocellular carcinoma progression by inhibiting CUL4A-mediated ubiquitination of LATS1. J Hematol Oncol 10: 91, 2017.
66. Zhang L, Li S, Choi YL, Lee J, Gong Z, Liu X, Pei Y, Jiang A, Ye M, Mao M, et al: Systematic identification of cancer-related long noncoding RNAs and aberrant alternative splicing of quintuple-negative lung adenocarcinoma through RNA-Seq. Lung Cancer 109: 21-27, 2017.

67. Jiang MM, Mai ZT, Wan SZ, Chi YM, Zhang X, Sun BH and Di QG: Microarray profiles reveal that circular RNA hsa circ_0007385 functions as an oncogene in non-small cell lung cancer tumorigenesis. J Cancer Res Clin Oncol 144: 667-674, 2018.

68. Chen Y, Yang Z, Deng B, Wu D, Quan Y and Min Z: Interleukin $1 \beta / 1 \mathrm{RA}$ axis in colorectal cancer regulates tumor invasion, proliferation and apoptosis via autophagy. Oncol Rep 43: 908-918, 2020

69. Zhao HQ, Dong BL, Zhang M, Dong XH, He Y, Chen SY, Wu B and Yang XJ: Increased KIF21B expression is a potential prognostic biomarker in hepatocellular carcinoma. World J Gastrointest Oncol 12: 276-288, 2020

70. Wolf D, Fiegl H, Zeimet AG, Wieser V, Marth C, Sprung S, Sopper S, Hartmann G, Reimer D and Boesch M: High RIG-I expression in ovarian cancer associates with an immune-escape signature and poor clinical outcome. Int J Cancer 146: 2007-2018, 2020.

71. Shiseki M, Ishii M, Miyazaki M, Osanai S, Wang YH, Yoshinaga K, Mori N and Tanaka J: Reduced PLCG1 expression is associated with inferior survival for myelodysplastic syndromes. Cancer Med 9: 460-468, 2020.

72. Sun Q, Hao Q and Prasanth KV: Nuclear long noncoding RNAs: Key regulators of gene expression. Trends Genet 34: 142-157, 2018.

73. Liu F, Cai Y, Rong X, Chen J, Zheng D, Chen L, Zhang J, Luo R, Zhao $\mathrm{P}$ and Ruan J: MiR-661 promotes tumor invasion and metastasis by directly inhibiting RB1 in non small cell lung cancer. Mol Cancer 16: 122, 2017.

74. Liang X, Li Z, Men Q, Li Y, Li H and Chong T: MiR-326 functions as a tumor suppressor in human prostatic carcinoma by targeting mucin1. Biomed Pharmacother 108: 574-583, 2018.

75. Li Y, Zheng F, Xiao X, Xie F, Tao D, Huang C, Liu D, Wang M, Wang L, Zeng F and Jiang G: CircHIPK 3 sponges miR-558 to suppress heparanase expression in bladder cancer cells. EMBO Rep 18: 1646-1659, 2017.

76. Li D, Hao X and Song Y: An integrated analysis of key microRNAs, regulatory pathways and clinical relevance in bladder cancer. Onco Targets Ther 11: 3075-3085, 2018

This work is licensed under a Creative Commons Attribution-NonCommercial-NoDerivatives 4.0 International (CC BY-NC-ND 4.0) License. 